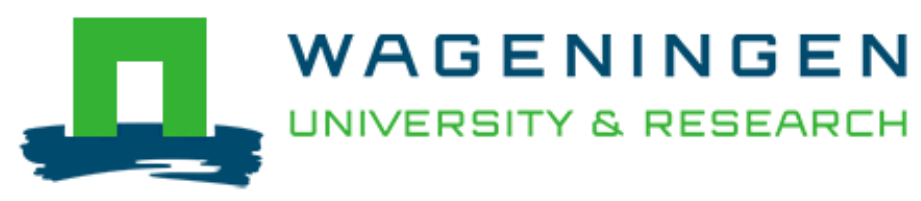

\title{
Estimating architecture-based metabolic scaling exponents of tropical trees using terrestrial LiDAR and 3D modelling
}

Lau, A., Martius, C., Bartholomeus, H., Shenkin, A., Jackson, T., Malhi, Y., ... Bentley, L. P.

This is a "Post-Print" accepted manuscript, which has been published in "Forest Ecology and Management"

This version is distributed under a non-commercial no derivatives Creative Commons (c) (1) $\Theta \Theta$ reproduction in any medium, provided the original work is properly cited and not used for commercial purposes. Further, the restriction applies that if you remix, transform, or build upon the material, you may not distribute the modified material.

Please cite this publication as follows:

Lau, A., Martius, C., Bartholomeus, H., Shenkin, A., Jackson, T., Malhi, Y., ... Bentley, L. P. (2019). Estimating architecture-based metabolic scaling exponents of tropical trees using terrestrial LiDAR and 3D modelling. Forest Ecology and Management, 439, 132-145. DOI: 10.1016/j.foreco.2019.02.019

You can download the published version at:

https://doi.org/10.1016/j.foreco.2019.02.019 
1 Estimating architecture-based metabolic scaling exponents of tropical trees using terrestrial LiDAR and 3D modelling

\footnotetext{
Alvaro Lau*1,2 ${ }^{*}$ Christopher Martius ${ }^{2}$, Harm Bartholomeus ${ }^{1}$, Alexander Shenkin ${ }^{3}$, Tobias Jackson $^{3}$, Yadvinder Malhi ${ }^{3}$, Martin Herold ${ }^{1}$, and Lisa Patrick Bentley ${ }^{4}$

${ }^{1}$ Laboratory of Geo-Information Science and Remote Sensing, Wageningen University and Research, Droevendaalsesteeg 3, 6708 PB, Wageningen, the Netherlands ${ }^{2}$ Center for International Forestry Research (CIFOR), P.O. Box 0113 BOCBD, Bogor 16000, Indonesia

${ }^{3}$ Environmental Change Institute, School of Geography and the Environment, South Parks Road, University of Oxford OX1 3QY, UK

${ }^{4}$ Department of Biology, Sonoma State University, 1801 East Cotati Avenue, Rohnert Park, CA 94928, USA
}

*corresponding author: Laboratory of Geo-Information Science and Remote Sensing, Wageningen University and Research, Droevendaalsesteeg 3, 6708 PB, Wageningen, the Netherlands e-mail: alvaro.lausarmiento@wur.nl 


\section{Abstract}

The geometric structure of tree branches has been hypothesized to relate to the mechanical safety and efficiency of resource transport within a tree. As such, the topology of tree architecture links physical properties within a tree and influences the interaction of the tree with its environment. Prior work suggests the existence of general principles which govern tree architectural patterns across of species and bio-geographical regions. In particular, West, Brown and Enquist (WBE; 1997) and Savage et al. (2010) derive scaling exponents (branch radius scaling ratio $\alpha$ and branch length scaling ratio $\beta$ ) from symmetrical branch parameters and from these, an architecture-based metabolic scaling rate $(\theta)$ for the whole tree. With this key scaling exponent, the metabolism (e.g., number of leaves, respiration, etc.) of a whole tree, or potentially a group of trees, can be estimated allometrically. Until now, branch parameter values have been measured manually; either from standing live trees or from harvested trees. Such measurements are time consuming, labour intensive and susceptible to subjective errors. Remote sensing, and specifically terrestrial LiDAR (TLS), is a promising alternative, being objective, scalable, and able to collect large quantities of data without destructive sampling. In this paper, we calculated branch length, branch radius, and architecture-based metabolic rate scaling exponents by first using TLS to scan standing trees and then fitting quantitative structure models (TreeQSM) models to 3D point clouds from nine trees in a tropical forest in Guyana. To validate these TLS-derived scaling exponents, we compared them with exponents calculated from direct field measurements of all branches $>10 \mathrm{~cm}$ at four scales: branch-level, cumulative branch order, tree-level and plot-level. We found a bias on the estimations of $\alpha$ and $\beta$ exponents due to a bias on the reconstruction of the branching architecture. Although TreeQSM scaling exponents predicted similar $\theta$ as the manually measured exponents, this was due to the combination of $\alpha$ and $\beta$ scaling exponents which were both biased. Also, the manually measured $\alpha$ and $\beta$ scaling exponents diverged from the WBE's theoretical exponents suggesting that trees in tropical environments might not follow the predictions for the symmetrical branching geometry proposed by WBE. Our study provides an alternative method to estimate scaling exponents at both the branch- and tree-level in tropical forest trees without the need for destructive sampling. Although this approach is based on a limited sample of nine trees in Guyana, it can be implemented for large-scale plant scaling assessments. These new data might improve our current understanding of metabolic scaling without harvesting trees.

\section{Keywords}

terrestrial LiDAR; WBE plant scaling exponent; quantitative structure models; architecture-based metabolic rate; destructive harvesting 


\section{Introduction}

Tropical forests are structurally complex ecosystems. This complexity is due to the distribution of woody stems and the three-dimensional arrangement of aboveground elements (i.e., leaves, branches, trunks) from the bottom to the top of the canopy (Saatchi et al., 2011). Detailed descriptions of the branching complexity of trees in forests can be traced back to Leonardo Da Vinci in the 15th century; however, it was not until the work of Francis Halle in the late 70's that tree form was qualitatively classified (Hallé et al., 1978). The architectural form of the tree is the result of a combination of both its genetic programme and its adaptive response to the surroundings (Hallé et al., 1978; Malhi et al. 2018) and influences physical (such as growth, water movement and nutrient allocation) and ecological processes (such as photosynthesis, $\mathrm{CO}_{2}$ sequestration and evapotranspiration) (Rosell et al., 2009). Indeed, similarities in relationships between physical and ecological processes suggest the existence of general principles underlying tree form (Savage et al., 2010; Sperry et al., 2012; Tredennick et al. 2013).

Several "universal" models, including the Geometric Similarity Model (McMahon and Kronauer, 1976), Stress Similarity model (Niklas, 1994) and the West, Brown and Enquist (WBE) model (West et al. 1997; West, 1999) have been developed to understand these principles with reproducible theoretical predictions (Tredennick et al., 2013). Among these, the WBE model (West et al., 1997; West, 1999) is the most tested and can be used to extrapolate the scaling of trees to larger spatial scales such as whole forests (West et al., 2009). The WBE model states that the scaling of metabolic rate and other biological functions has its origin in a (theoretical) optimal branching system network with both internal (vascular) and external (branching) components (West et al., 1997; West, 1999; Savage et al. 2010; see Appendix A.1 for more information regarding WBE model). Moreover, an idealized branching network which must be symmetrical, self-similar and hierarchical is assumed for the external structure of trees in the WBE model (Appendix A.1). From this branching network, three key parameters (branching ratio, branch radius and branch length) can be extracted and used to estimate scaling exponents (West et al., 1997; West, 1999; Savage et al., 2010). While the WBE model has been criticized since, real tree branches rarely conform to idealized branching networks, a recent study by Brummer et al. (2017) showed that showed that asymmetric branching can incorporated into the WBE model and does not drastically change predictions.

To apply the WBE model to forest modelling and management across spatial scales, an accurate quantification of the trees external branching architecture is needed. However, few studies quantitatively assess branch architecture at the branch- or tree-level within the context of plant scaling models (Nygren and Pallardy, 2008; Bentley et al., 2013; Tredennick et al., 2013). Furthermore, these studies use either destructive harvesting or direct measurements and the intensity of manual labour required to sample large quantities of trees with enough detail have been a bar to progress in this field (Bentley et al. 2013). Large trees $(\mathrm{DBH}>70 \mathrm{~cm})$ are hardly ever measured manually (due to the intensity of manual labour) and most of the tests within the context of plant scaling models are based on small trees. Additionally, manual measurements require subjective decisions, such as defining where a branch starts and finishes, which may limit their usefulness in plant scaling models. As encountered by Lau et al. (2018), their results found a bias in the branch length measurements. This because the "branch length" definition differed between the manual measurements and TreeQSM. An accurate estimation and quantification of external branching architecture is key to understand the linkage of plant form and function across multiple spatial scales.

Terrestrial Light Detection and Ranging (LiDAR) or terrestrial laser scanning (TLS) is a valuable tool to capture the three-dimensional structure of trees and, in combination with specialized algorithms, to assess the woody structure in a repeatable, non-invasive and objective way (Wilkes et al. 2017; Malhi et al., 2018). This active remote sensing technique is based on the emission and reception of tens to hundreds of thousands of mono-spectral laser beam pulses (Grau et al., 2017) which are propagated into the surroundings of the instrument up to hundreds of metres (Malhi et al., 2018). When these pulses hit an object they are reflected back to the instrument. The reflected pulse's return time is used to create an accurate and highly detailed spatial three-dimensional representation of the 
surface of the objects surrounding the scanner. With the use of specialized software, a highly detailed 3D point cloud of the scanned area is created (Wilkes et al., 2017).

TLS is increasingly used to extract various attributes from scanned forests. Initial studies focused on extracting plot-level attributes of trees (Côté et al., 2012; van Leeuwen et al., 2011; Dassot et al. 2011; Newnham et al., 2015; Xi et al., 2016; Wilkes et al., 2017; Grau et al., 2017), mostly due to the intensive manual labour required to identify and extract individual trees from the massive point cloud. The development of tree segmentation algorithms (Raumonen et al., 2015; Ayrey et al., 2017) assisted in a semi-automated extraction of individual trees and allowed for tree-level measurements to be collected. Moreover, the development of quantitative models to reconstruct the fine structure of trees (e.g. TreeQSM; Raumonen et al., 2013 and Simple Tree; Hackenberg et al., 2015) further refined approaches available to derive indirect quantitative parameters related to tree architecture. In particular, using these algorithms and quantitative structure models, branch diameter (Tansey et al. 2009, Huang et al., 2011), tree height (Burt et al., 2013, Krooks et al., 2014; Brede et al., 2017), and crown diameter and area (Zhao et al., 2012; Srinivasan et al., 2015) can be automatically estimated from individual tree point clouds.

Reconstruction algorithms also have the potential to estimate tree volume, and indirectly, aboveground biomass (Calders et al., 2015; Gonzalez de Tanago et al., 2017; Momo Takoudjou et al., 2018; Saarinen et al., 2017; Stovall et al., 2017). Further, from estimations of volume and biomass, allometric models can be constructed Olagoke et al. (2016). Other characteristics of trees, such as root structure (Smith et al. 2014a; Paynter et al., 2016) and species recognition (Ákerblom et al., 2017) can also be extrapolated from TLS scans. TLS scanning of the same area at different periods allowed Olivier et al. (2017) to observe canopy change and Kaasalainen et al. (2014) to observe aboveground biomass change. The reconstruction of tree structure in fine detail allows not only the quantification of tree productivity, as mentioned above, but also the assessment of tree structure from an ecological point of view. Malhi et al. (2018) detailed the potential application of TLS and quantitative structure models to understand the ecological challenges regarding branching architecture, surface area scaling, tree respiration, seed dispersal and tree mechanics.

As detailed above, TLS scans are a promising alternative to collect large quantities of data without destructive sampling and subjective bias over various spatial and temporal scales. While TLS has a variety of ecological applications for forest modelling, this study aims to provide a better basis for understanding metabolic scaling through an approach to estimate scaling exponents using TLS and TreeQSM (Fig. 1). Importantly, with this research, we do not try to revise the theory of metabolic scaling, but rather, propose and validate a methodological approach to estimate model parameters that does not rely on destructive sampling and increases data collection efficiency compared to traditional methods. Moreover, we focused our methodological approach on tropical trees. TLS Scanning and 3D modelling the complex external architecture of tropical trees in-situ adds challenge to our research (Wilkes et al., 2017; Lau et al., 2018). To date, no study has used remote sensing estimates of branch parameter values to estimate $\alpha$ and $\beta$ exponents. In this paper we aim to: (i) estimate WBE model scaling exponents from TLS point clouds and TreeQSM; (ii) validate these exponent estimates from the TreeQSM with manually measured exponent estimates and; (iii) assess if theoretical metabolic scaling predictions are included within our estimations.

\section{Material and methods}

\subsection{Study area}

Field data were acquired from Vaitarna Holding's concession, central Guyana, during November 2014 (see Lau et al. 2018 for details). The area is a lowland tropical moist forest with an elevation of $117 \mathrm{~m}$ above sea level and a mean rainfall of $2195 \mathrm{~mm} \mathrm{yr}^{-1}$. Seven Eperua grandiflora, one Ormosia coutinhoi, and one Eperua falcata (See Fig. A.1) had been already marked for logging in the forest management plan of the concession (for timber production or management purposes). The diameter at breast height $(\mathrm{DBH})$ across all trees ranged from $61.3 \mathrm{~cm}$ to $97.0 \mathrm{~cm}$ and the height ranged from $18.8 \mathrm{~m}$ 

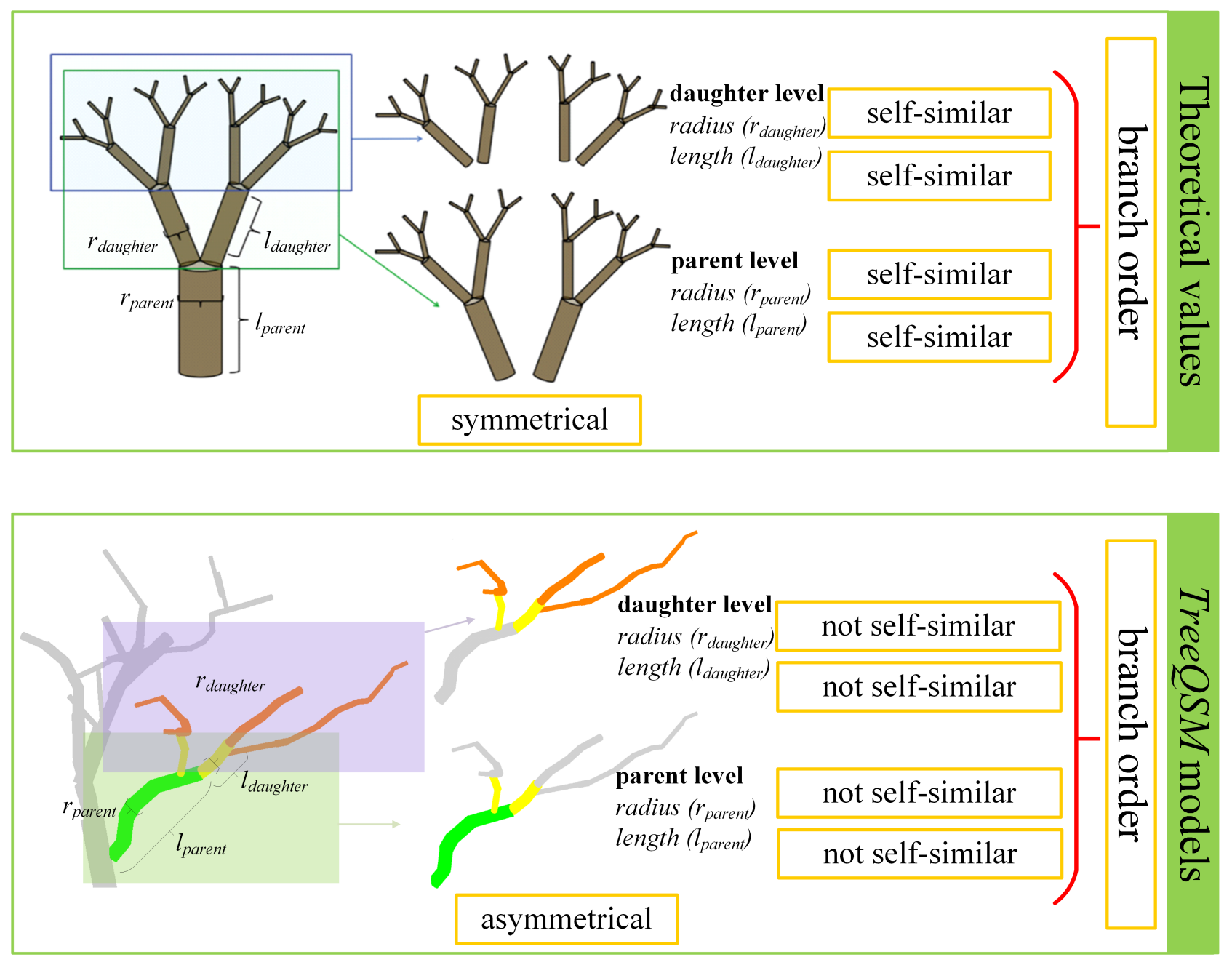

Figure 1: Branch scaling ratios for idealized symmetrical trees (top) and for trees modelled with TreeQSM (bottom) based on branch radius $(r)$, length $(l)$, and branching ratio $(n)$. The branching ratio is the number of daughter branches per parent branch. The branch radius scaling parameter is $\frac{r_{\text {daughter }}}{r_{\text {parent }}}$ and the length scaling parameter is $\frac{l_{\text {daughter }}}{l_{\text {parent }}}$. Based on Fig. 1 from Bentley et al. (2013) and Fig. 3 from Malhi et al. (2018). Refer to digital version for colour image.

to $29.9 \mathrm{~m}$. A $30 \times 40 \mathrm{~m}$ plot was set up around each selected tree in the expected felling direction. We scanned each plot with TLS, harvested the tree and took detailed geometrical measurements of each branch $>10 \mathrm{~cm}$ diameter. Plot details can be found in Gonzalez de Tanago et al. (2017) and Wilkes et al. (2017).

\section{$2.2 \quad$ TLS acquisition and plot design}

All TLS datasets were acquired using a RIEGL VZ-400 V-Line 3D terrestrial laser scanner (RIEGL Laser Measurement Systems GmbH, Horn, Austria, www.riegl.com). The instrument used in this study is a discretized multiple-return LiDAR scanner with a $1550 \mathrm{~nm}$ wavelength and a $0.35 \mathrm{mrad}$ beam divergence (Gonzalez de Tanago et al., 2017; Wilkes et al., 2017). This TLS has a scan range of $360^{\circ}$ in the azimuth, $100^{\circ}$ in the zenith and the angular resolution used in this study was $0.06^{\circ}$. In each plot, 9 to 16 scan positions were set up and 80 to 1005 -cm-diameter cylindrical reflecting targets (tie-points) were distributed evenly in the plot. The tie-points were placed in such a way that each of them could be scanned from several positions. These tie-points were later used to co-register the 
individual points clouds into a unified point cloud as in Gonzalez de Tanago et al. (2017); Wilkes et al. (2017) and Lau et al. (2018).

\subsection{Manual measurements of branches}

The manual measurements of the nine harvested trees were analysed in Lau et al. (2018). Here, we give a summary of the methodology employed. We measured a total of 279 individual branches up to $10 \mathrm{~cm}$ diameter with $1 \mathrm{~cm}$ resolution forestry tape. We took two measurements of each branch: length $(\mathrm{m})$ and diameter $(\mathrm{cm})$. The length was defined as the distance between the base and the termination of the branch and the diameter was defined as the average of two diameter measurements, one taken at the base, and the other, at the termination of the branch (See Fig. 1 in Lau et al., 2018). Finally, we defined the branch order and hierarchy. The branch order was established "centrifugally", starting from the main stem and adding an order at every branch node. The branch hierarchy was defined as the branch correspondence between a parent branch and daughter branch. A daughter branch is any branch with originates from a parent branch and the parent branch was recorded for each individual branch.

\subsection{Branching reconstruction}

The branching reconstruction of the scanned trees was performed in Lau et al. (2018) and had three components: (a) manual tree extraction from the point cloud (Fig. 2 a). All individual TLS scans were co-registered into a plot point cloud, in which the harvested trees were located and extracted. For quality control, visual inspection was performed on each tree point cloud to ensure that no parts of the tree were missing; (b) 3D reconstruction of individual tree point clouds using TreeQSM (Figs. 2b-c and Raumonen et al. 2013 and Fig. 2 in Lau et al., 2018). A series of steps was performed to ensure that the seven best-fitted TreeQSM models were obtained (Lau et al., 2018); and, (c) comparison of TreeQSM branches and manually measured branches (Fig. $2 \mathrm{~d}$ ). For this step, each manually measured branch was visually paired with a QSM modelled branch following the structure of the modelled tree. If a measured branch did not have a modelled branch, the measured branch was not paired and excluded from further analysis. If a measured branch corresponded to two or more modelled branches, we quantitatively analysed the similarity of these branches using their length and diameter. We used a diagonal-norm approach to standardize both parameters and analysed their similarities. The modelled branch most similar to the measured branch was chosen as the best-fitted pair.

The geometrical structure was determined as follows: TreeQSM branch length was the sum of the length of all cylinders of the same branch, TreeQSM branch diameter was the average of the first and last cylinder of the same branch, and branch order was estimated starting from the main stem and adding a new level at each branch node.

\section{$2.5 \quad$ Tree metrics}

Tree architecture was analysed in Lau et al. (2018) and a summary of tree metrics for this dataset can be seen in Table 1. Lau et al. (2018) validated the reconstruction accuracy of branches lengths, branches diameters and branch orders of 279 modelled branches compared with manually measured branches. Their method found and reconstructed $95 \%$ of branches thicker than $30 \mathrm{~cm}$ diameter. The accuracy of the length and diameter of the modelled branches varied among diameter classes. For branches smaller than $50 \mathrm{~cm}$ in diameter, the length of the modelled branches was underestimated by $20 \%$.

For branches greater than $50 \mathrm{~cm}$ in diameter, the length of the modelled branches was overestimated by $1 \%$. For branches between $10 \mathrm{~cm}$ and $20 \mathrm{~cm}$ in diameter, the modelled branch diameters were overestimated by $40 \%$. For branches with a diameter between $20 \mathrm{~cm}$ and $60 \mathrm{~cm}$, diameter was underestimated by $8 \%$; if the branch diameter was greater than $60 \mathrm{~cm}$, diameter was underestimated by $6 \%$. In this study, the branch order was correctly assigned with an overall accuracy of $99 \%$. 

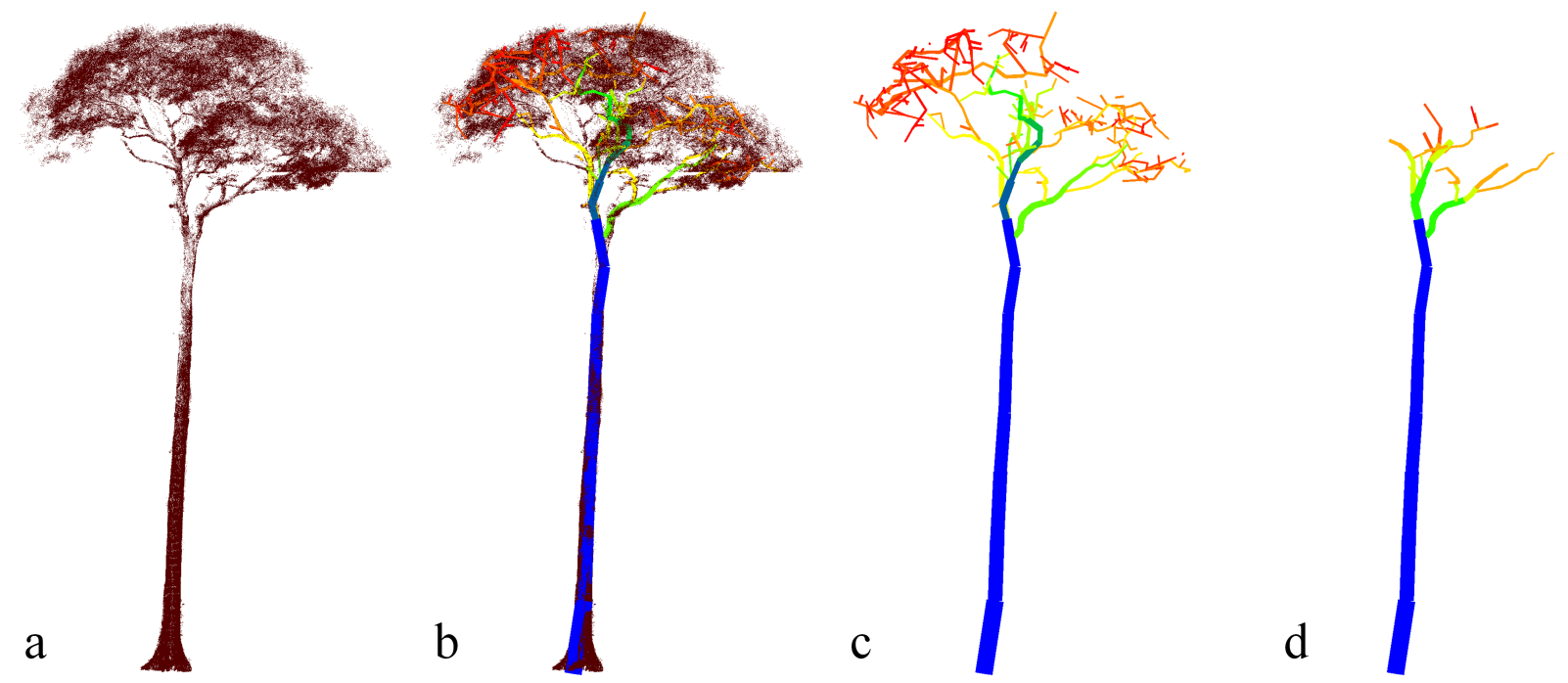

Figure 2: (a) Ormosia coutinhoi tree point cloud, (b) TreeQSM with branches $>10 \mathrm{~cm}$ diameter reconstructed along with the tree point cloud, (c) QSM branches classified by branch order and (d) QSM branches which were paired with manually measured branches. Refer to digital version for colour image.

Table 1: Tree metrics from TreeQSM branches and manually measured branches from the Vaitarna dataset (Lau et al., 2018).

\begin{tabular}{|c|c|c|c|c|c|c|}
\hline \multirow{2}{*}{$\begin{array}{l}\text { Diameter } \\
\text { class }(\mathrm{cm})\end{array}$} & \multirow{2}{*}{$\begin{array}{c}\text { Measured } \\
\text { branches }\end{array}$} & \multirow{2}{*}{$\begin{array}{c}\text { Reconstruction } \\
\text { Accuracy (\%) }\end{array}$} & \multicolumn{2}{|c|}{ Absolute error } & \multicolumn{2}{|c|}{ Relative error } \\
\hline & & & Length $(\mathrm{m})$ & Diameter $(\mathrm{cm})$ & Length (\%) & Diameter (\%) \\
\hline $10-20$ & 160 & 45 & $-1.03 \pm 1.81$ & $5.14 \pm 5.50$ & 12 & 40 \\
\hline $20-30$ & 67 & 67 & $-0.67 \pm 1.50$ & $-0.65 \pm 4.76$ & 10 & -2 \\
\hline $30-40$ & 26 & 84 & $-0.42 \pm 2.19$ & $-5.33 \pm 5.26$ & 37 & -15 \\
\hline $40-50$ & 11 & 92 & $-0.21 \pm 1.37$ & $-4.23 \pm 7.83$ & 19 & -9 \\
\hline $50-60$ & 7 & 100 & $-0.10 \pm 0.76$ & $-3.61 \pm 8.98$ & -1 & -7 \\
\hline $60-70$ & 5 & 100 & $0.34 \pm 0.40$ & $-5.33 \pm 5.68$ & 3 & -9 \\
\hline$\geq 70$ & 3 & 100 & $0.39 \pm 0.30$ & $-1.54 \pm 0.80$ & 2 & -2 \\
\hline
\end{tabular}

\subsection{Estimation of WBE scaling exponents}

Based on previous work by Savage et al. (2010) and Bentley et al. (2013), the scaling exponents from the WBE model for idealized trees can be described using three key parameters (West, 1999, Malhi et al. 2018): branch radius scaling ratios $\left(\alpha_{\text {branch }}\right)$, branch length scaling ratios $\left(\beta_{\text {branch }}\right)$, and branching ratios ( $n$, ratio between number of daughter branches per parent branch). From these branch-level attributes, the scaling of architecture-based metabolic rate $\left(\theta_{\text {branch }}\right)$ can be further predicted (Table 2). Within the WBE model, constant values are given to these parameters when idealized trees are estimated $(\alpha=1 / 2, \beta=1 / 3$, and $\theta=0.69$; West, 1999; Savage et al., 2008, 2010). We used $\theta=0.69$ and not the WBE prediction of $\theta=3 / 4$ to concord with the predictions for finite-size effects from restricting size range of plants (Savage et al. 2010). Moreover, (von Allmen et al. 2012 ) also found a lower value of $\theta(0.62 \pm 0.016)$ and Brummer et al. (2017) in their work on asymmetric branching also found that $\theta$ ranged between 0.5 and 1 in asymmetric branching.

\subsection{Assessment of WBE scaling exponents}

The scaling exponents $\alpha, \beta$, and $\theta$ were assessed at different levels: 
Table 2: Scaling exponents $\alpha_{\text {branch }}$ and $\beta_{\text {branch }}$ were calculated at branch-level and definitions are as follows: $r=$ branch radius, $n=$ number of branches, and $l=$ branch length, while the $\theta_{\text {branch }}$ was derived from $\alpha_{\text {branch }}$ and $\beta_{\text {branch }}$.

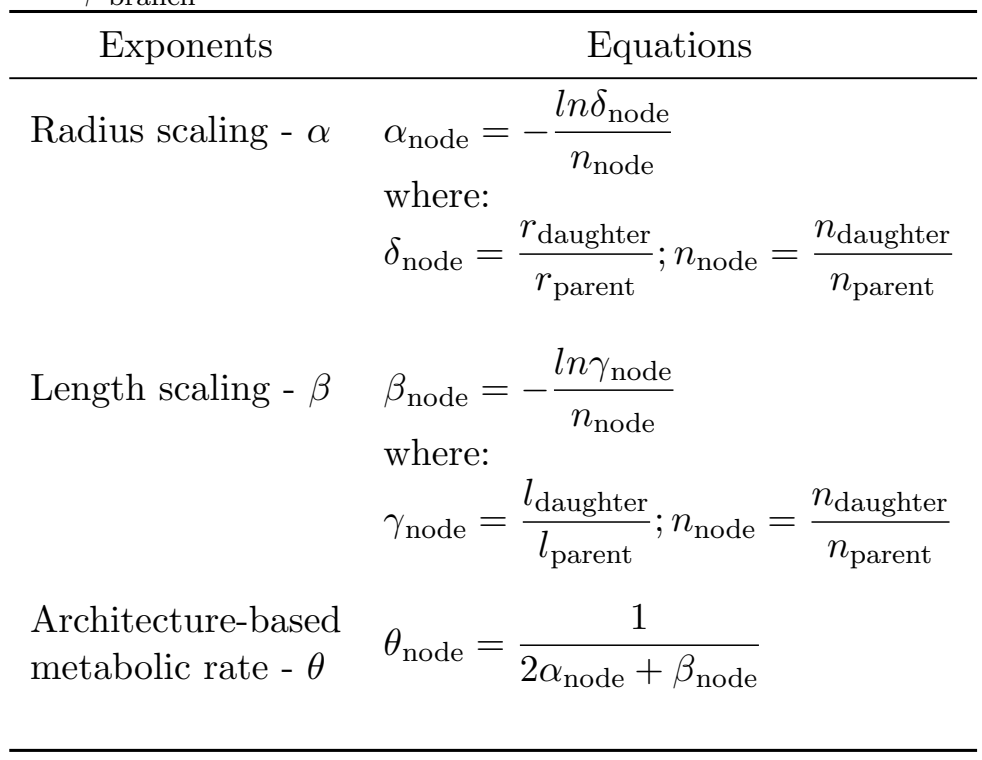

- Branch-level: scaling exponents at branch-level are shown as the distribution of the exponents, calculated from all branch nodes as in Table 2 and Fig. 1 of all the branch nodes assessed in this study. We estimated the median exponents, $95 \%$ confidence interval (CI), the bias (in \%), and the significant differences between TreeQSM and manually measured scaling exponents for all branches.

- Cumulative branch order: scaling exponents at branch-level were calculated from $2^{\text {nd }}$ to $8^{\text {th }}$ cumulative branch order and are shown as the median exponents and $95 \%$ CI of all the branch nodes per cumulative branch order. We assessed the bias (in \%) and the significant differences between TreeQSM and manually measured scaling exponents per cumulative branch order.

- Tree-level: scaling exponents at tree-level are shown as the median exponents and $95 \%$ CI of all the branch nodes within a tree. We assessed the bias (in \%) and the significant differences between TreeQSM and manually measured scaling exponents per tree.

- Plot-level: scaling exponents at plot-level are shown as the median ranges among the trees in this study.

First, we tested for normality of the data distribution with a Shapiro-Wilks test. Then, and depending on the distributional assumption, we estimated the median with $95 \%$ CI or (pseudo) median with $95 \%$ CI from Wilcoxon signed-rank test. Median exponents were used instead of the arithmetic mean because we did not want to assume unimodal and symmetrical distributions and could not use the geometric mean due to negative numbers (Bentley et al., 2013). We assessed the bias as the deviation (in \%) of the TreeQSM scaling exponents from the manually measured scaling exponents. Finally, to test the significant differences between the TreeQSM and manually measured scaling exponents, we used either a paired t-test or Wilcoxon signed-rank test, also depending on the distributional assumption. We included the theoretical predictions and analysed whether the theoretical predictions fall within the CI of our estimations in all three levels. 


\section{Results}

\subsection{Scaling exponents from TreeQSM branching reconstruction}

The accuracy of the tree metrics used in this paper (Table 1) influenced the estimations of the WBE exponents. The underestimation of the TreeQSM branch radius parameter and the overestimation of the TreeQSM branch length parameter displayed in Table 1 can also be seen as a bias in the $\alpha$ and $\beta$ exponents (Fig. 3 and Table 3) and the cumulative exponents (Fig. 4 and Table A.2).

The average underestimation of the radius parameter from the branching reconstruction incurs in a negative bias towards the TreeQSM $\alpha_{\text {branch }}$ (Table 3) and the cumulative $\alpha_{\text {branch }}$ (Table A.2). Likewise, the great overestimation of the length parameter incurs in a great positive bias towards the TreeQSM $\beta_{\text {branch }}$ (Table 3) and the cumulative $\beta_{\text {branch }}$ (Table A.2). Since the estimation of $\theta$ is computed from $\alpha$ and $\beta$ exponents, the two biases have a direct influence over the estimation of $\theta_{\text {branch }}$.

\subsection{Scaling exponents at branch-level}

The scaling exponent distributions at branch-level of $\alpha_{\text {branch }}, \beta_{\text {branch }}$ and $\theta_{\text {branch }}$ for both, TreeQSM and manually measured branches were not normally distributed $(p$-value $<0.05$, Fig. 3, and Table A.1). 
Figure 3: Distribution of individual branches for $\alpha_{\text {branch }}$ (top), $\beta_{\text {branch }}$ (middle) and $\theta_{\text {branch }}$ (bottom) exponents as density function (y-axis), for TreeQSM and manually measured scaling exponents. Vertical dashed line indicates WBE idealized predictions for $\alpha=1 / 2, \beta=1 / 3$ and $\theta=0.69$. Refer to digital version for colour image.

\section{Radius scaling exponent (*)}

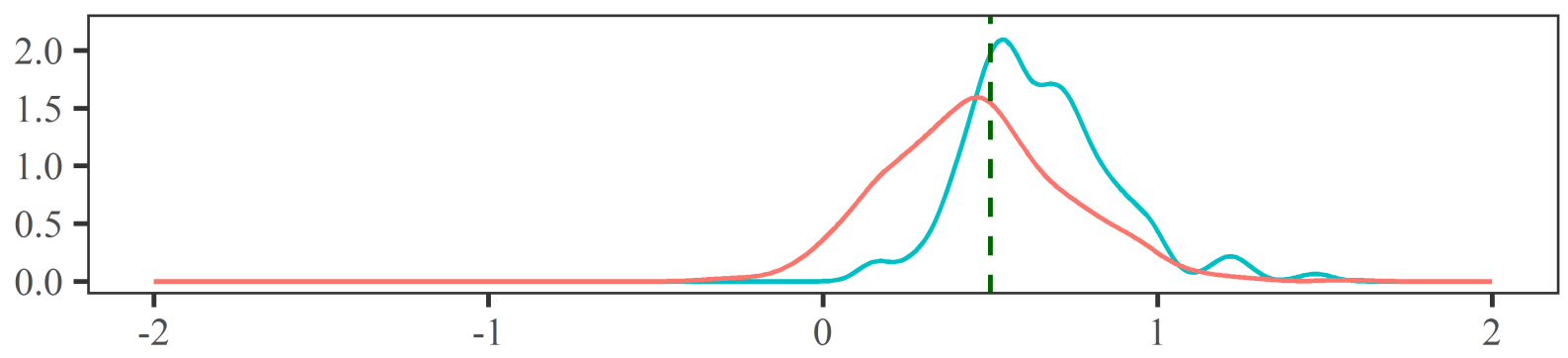

\section{Length scaling exponent (*)}

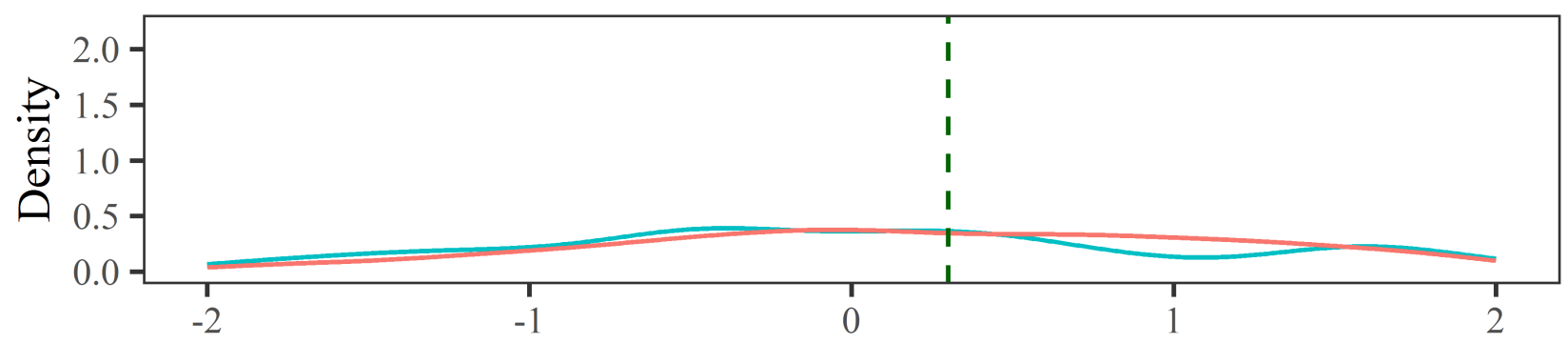

Architecture-based metabolic rate ( $\dagger)$

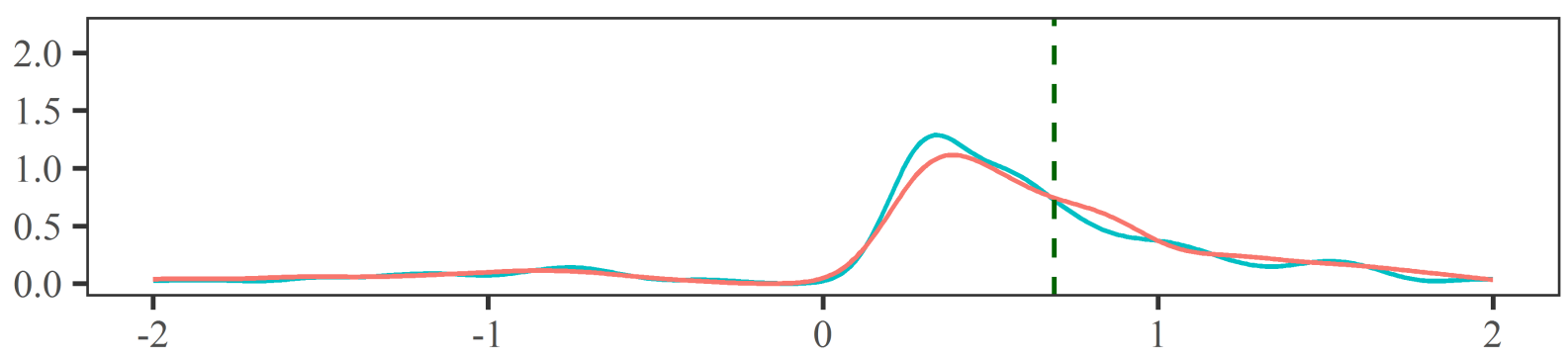

— TreeQSM branch — Manually measured branch

* significant different at 0.05 probability level.

$\dagger$ non significant different at 0.05 probability level.

The (pseudo) median exponents, 95\% CIs, bias, and the significant differences for scaling exponent distributions at branch-level are displayed in Table 3. The TreeQSM $\alpha_{\text {branch }}$ showed a lower pseudo(median) and a bias of $-29 \%$ than its manually measured counterpart. However, the TreeQSM

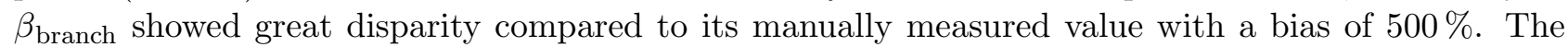
TreeQSM $\theta_{\text {branch }}$ showed a higher pseudo(median) and a bias of $15 \%$ than its manually measured counterpart. We compared TreeQSM and manually measured exponents and found a significant difference $(p$-value $<0.05)$ for $\alpha_{\text {branch }}$ and $\beta_{\text {branch }}$; but not for $\theta_{\text {branch }}$ exponents (Table 3). Likewise, 
when comparing the $95 \%$ CIs, we found that the CIs of $\alpha_{\text {branch }}$ and $\beta_{\text {branch }}$ showed disparity; while the CI of $\theta_{\text {branch }}$ overlapped (Table 33).

Table 3: Scaling exponents of $\alpha_{\text {branch }}(n=484), \beta_{\text {branch }}(n=484)$, and $\theta_{\text {branch }}(n=484)$ of the TreeQSM and manually measured branches at branch-level. Exponents shown as (pseudo) median with $95 \%$ CI for the branch-level distribution and exponents are shown as average. Bias (\%) and significant differences were calculated between TreeQSM and manually measured scaling exponents.

\begin{tabular}{|c|c|c|c|c|c|c|c|}
\hline \multirow{2}{*}{$\begin{array}{l}\text { Scaling } \\
\text { exponents }\end{array}$} & \multirow{2}{*}{$\begin{array}{c}\text { Theoretical } \\
\text { class }\end{array}$} & \multicolumn{2}{|c|}{ TreeQSM } & \multicolumn{2}{|c|}{ Manually measured } & \multirow[t]{2}{*}{ Bias $(\%)$} & \multirow[t]{2}{*}{ Signif. } \\
\hline & & (pseudo) median & CI $(95 \%)$ & (pseudo) median & CI $(95 \%)$ & & \\
\hline$\alpha_{\text {branch }}$ & 0.50 & 0.45 & $0.43-0.48$ & 0.63 & $0.62-0.65$ & -29 & $*$ \\
\hline$\beta_{\text {branch }}$ & 0.33 & 0.42 & $0.31-0.54$ & 0.07 & $-0.06-0.2$ & 500 & $*$ \\
\hline$\theta_{\text {branch }}$ & 0.69 & 0.59 & $0.53-0.65$ & 0.50 & $0.4-0.56$ & 18 & $\dagger$ \\
\hline
\end{tabular}

* significant different at 0.05 probability level.

$\dagger$ non significant different at 0.05 probability level.

\subsection{Branch scaling exponents per cumulative branch order at branch-level}

We analysed the representation of the scaling exponents for cumulative branch orders for both, TreeQSM and manually measured branches up to the $8^{\text {th }}$ branch order (Fig. 4 and Table A.2). The TreeQSM and manually measured scaling exponents followed the same pattern in each cumulative scaling exponent (Fig. 4). For the cumulative $\alpha_{\text {branch }}$, the TreeQSM exponents had lower (pseudo) median exponents, a negative bias, and a significant difference from the $3^{\text {rd }}$ branch order onwards (Fig. 4 and Table A.2). For the cumulative $\beta_{\text {branch }}$ and cumulative $\theta_{\text {branch }}$, both TreeQSM exponents had higher (pseudo) median exponents than the measured ones. However, cumulative $\beta_{\text {branch }}$ showed great disparity (great bias and significance) from the $4^{\text {th }}$ branch order onwards. Cumulative $\theta_{\text {branch }}$ showed low bias and differences were not significant different across all orders. 
Figure 4: Cumulative (pseudo) median and $95 \%$ CI for $\alpha_{\text {branch }}$ (top), $\beta_{\text {branch }}$ (middle) and $\theta_{\text {branch }}$ (bottom) exponents for TreeQSM and manually measured branches up to $8^{\text {th }}$ cumulative branch order. The $95 \%$ CIs are shown as vertical lines on the (pseudo) median exponents. Horizontal dashed line indicates WBE idealized predictions for $\alpha=1 / 2, \beta=1 / 3$ and $\theta=0.69$. Refer to digital version for colour image.

\section{Radius scaling exponent}

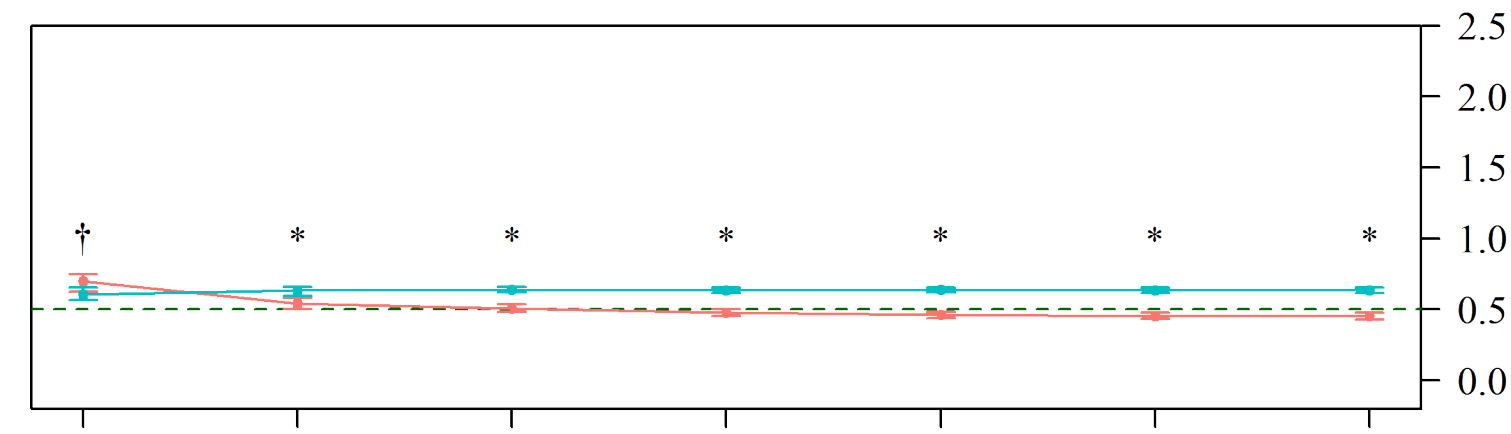

up to 2 nd up to 3 rd up to 4th up to 5th up to 6th up to 7 th up to 8 th

\section{Length scaling exponent}

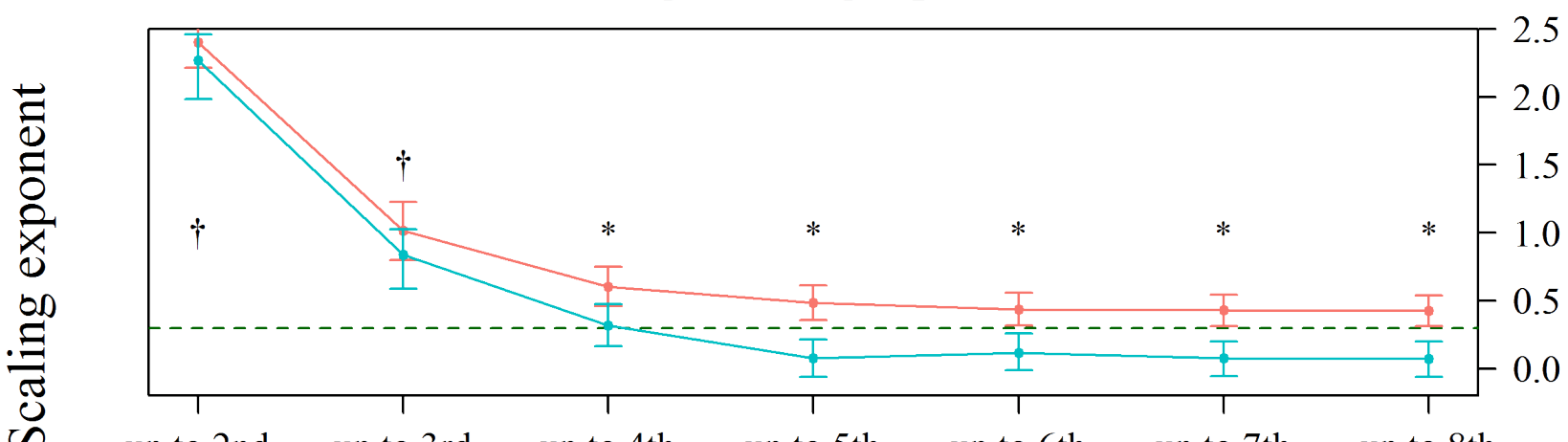

up to 2nd up to 3rd up to 4th up to 5th up to 6th up to 7th up to 8th

Architecture-based metabolic rate

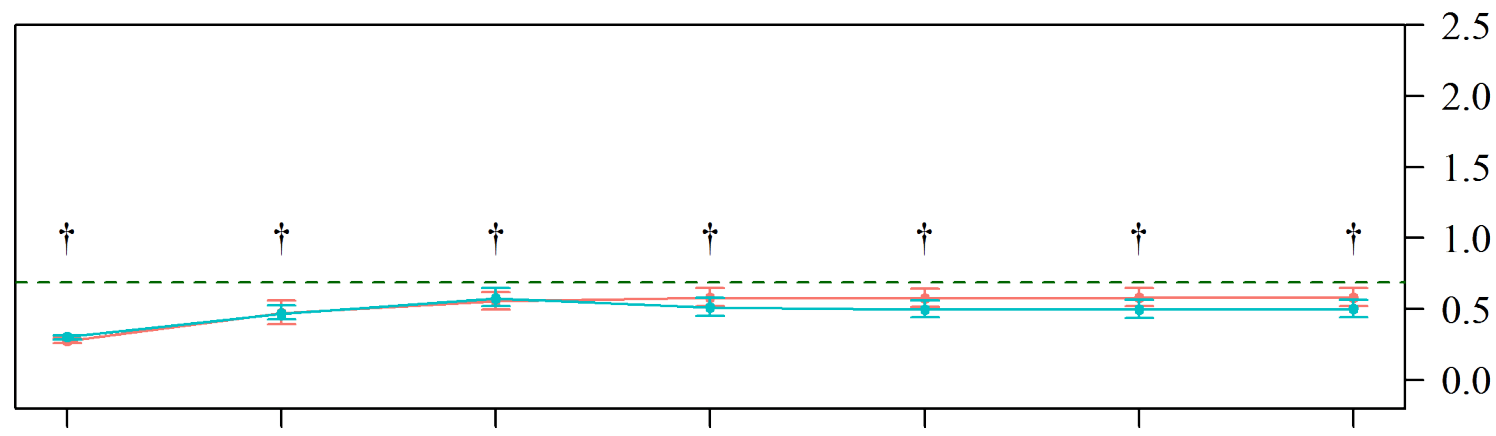

up to 2nd up to 3rd up to 4th up to 5th up to 6th up to 7th up to 8th

— TreeQSM branches _- $\begin{gathered}\text { Manually } \\ \text { measured branches } \quad-- \text { Theoretical values }\end{gathered}$

\section{Cumulative branch order}

* significant different at 0.05 probability level.

$\dagger$ non significant different at 0.05 probability level. 
Cumulative $\alpha_{\text {branch }}$ showed no overlapping CIs and significant differences for cumulative branch order higher than $3^{\text {rd }}$ order (Fig. 4 and Table A.2). The cumulative $\beta_{\text {branch }}$ displayed a significant variation and non-overlapping CIs from the $4^{\text {th }}$ cumulative branch order onwards. On the other hand, cumulative $\theta_{\text {branch }}$ showed overlapping CIs and no significant differences across all orders.

\subsection{Scaling exponents at tree-level and plot-level}

Figure 5 and Table A.3 shows the (pseudo) median exponents, the $95 \%$ CI, bias (\%), and the significant difference for each exponent for each tree, and ranges for plot-level. The average (pseudo) median exponents for the TreeQSM and manually measured exponents were 0.46 and 0.64 for $\alpha_{\text {tree }}, 0.41$ and 0.05 for $\beta_{\text {tree }}$, and 0.56 and 0.51 for the $\theta_{\text {tree }}$, respectively. 
Figure 5: (pseudo) median exponents, $95 \%$ CI, and significant difference for $\alpha_{\text {tree }}$ (top), $\beta_{\text {tree }}$ (middle) $\theta_{\text {tree }} 9$ bottom) for TreeQSM and manually measured individual trees. The $95 \%$ CIs are shown as vertical lines on the (pseudo) median exponents. Horizontal dashed line indicates WBE idealized predictions for $\alpha=1 / 2, \beta=1 / 3$ and $\theta=0.69$. Plot-level figures display ranges among the trees' (pseudo) median exponents. Refer to digital version for colour image.
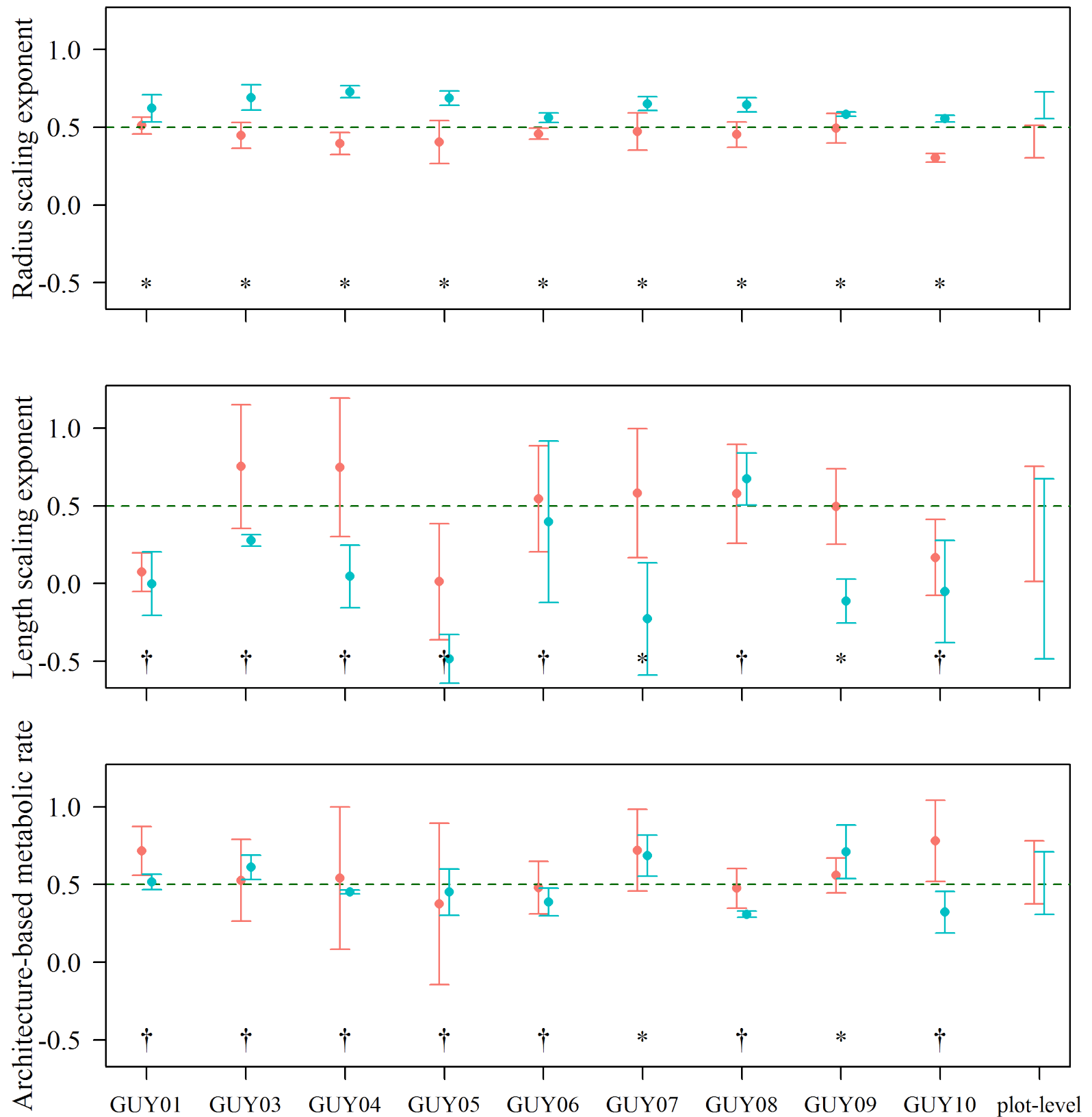

- TreeQSM tree

— Manually measured tree - - Theoretical values

* significant different at 0.05 probability level.

$\dagger$ non significant different at 0.05 probability level. 
When comparing TreeQSM with manually measured trees the $\alpha_{\text {tree }}$ and $\theta_{\text {tree }}$, both predictions followed similar patterns. As with the scaling exponents at cumulative branch-level (Fig. 4), TreeQSM $\alpha_{\text {tree }}$ consistently underestimated the manually measured $\alpha_{\text {tree }}$ with a range of bias between $-38 \%$ to $-15 \%$. In contrast, TreeQSM $\beta_{\text {tree }}$ exponents overestimated manually measured $\beta_{\text {tree }}$ and showed great disparity with a bias between $-426 \%$ to $1027 \%$. As a result, TreeQSM $\theta_{\text {tree }}$ exponents were higher than the manually measured $\theta_{\text {tree }}$, with a bias between $-36 \%$ to $101 \%$.

At plot-level, the range of TreeQSM $\alpha_{\text {plot }}$ (pseudo) median exponents did not overlap and underestimated the manually measured $\alpha_{\text {plot }}$ (Fig. 5 and Table A.3). For the TreeQSM $\beta_{\text {plot }}$, the range greatly overlap and slightly overestimated the manually measured $\beta_{\text {plot }}$. In the same way, for TreeQSM $\theta_{\text {plot }}$, the range of exponents greatly overlap and slightly overestimated the manually measured $\theta_{\text {plot }}$.

\subsection{Theoretical scaling exponents inclusion}

At branch-level, the $95 \%$ CI ranges for manually measured $\alpha_{\text {branch }}, \beta_{\text {branch }}$, and $\theta_{\text {branch }}$ excluded the theoretical exponents (Table 3). The manually measured $\alpha_{\text {branch }}$ range was higher than the theoretical $\alpha(0.5)$. On the contrary, manually measured $\beta_{\text {branch }}$ and $\theta_{\text {branch }}$ ranged were lower than the theoretical $\beta(0.33)$ and $\theta(0.69)$, respectively. For TreeQSM, only $\alpha_{\text {branch }}$ and $\theta_{\text {branch }}$ ranges excluded the theoretical exponents. The TreeQSM $\beta_{\text {branch }}$ included the theoretical exponents within its range. The TreeQSM ranges for $\alpha_{\text {branch }}$ and $\theta_{\text {branch }}$ were lower than the theoretical $\alpha(0.5)$ and $\theta$ (0.69), respectively.

At the cumulative branch order, most of the cumulative branch orders for both, TreeQSM and manually measured cumulative exponents excluded the theoretical values from their $95 \%$ CI ranges (Fig. 4 and Table A.2). At tree-level, most of the TreeQSM trees included the three theoretical exponents within their $95 \%$ CI ranges (Fig. 5 and Table A.3). For the manually measured trees, most of the trees included theoretical $\beta$ and $\theta$ (and excluded $\alpha$ ) within their 95\% CI ranges. At plot-level, the theoretical exponents were included within the ranges of all scaling exponents from TreeQSM and manually measured exponents except for the manually measured $\alpha_{\text {plot }}$ (Table A.3). The range for $\alpha_{\text {plot }}$ was higher than the theoretical value.

\section{Discussion}

\subsection{Constraints in the branch architecture}

This study generated the first quantitative measurements of metabolic scaling exponents from the WBE model using 3D models from point clouds of tropical trees. Our results show that, with some limitations, radius, length and architecture-based metabolic rate scaling exponents can be derived from 3D data of tree point clouds. Importantly, there is some error in these estimates as we observed systematic deviations between TreeQSM modelled and measured measurements of branch architecture. The large divergence in the $\beta_{\text {branch }}$ ratios was caused by the large absolute length error between the TreeQSM estimates and the manual measurements (Table 1). For branches greater than $50 \mathrm{~cm}$, the length of TreeQSM branches was overestimated by $1 \%$ and, for branches thinner than $50 \mathrm{~cm}$, the average length of TreeQSM branches was underestimated by $20 \%$ (See Table 1). As found in Lau et al. (2018), this bias is likely due to a conceptual difference in the definition of the point of branch termination between TreeQSM and manual measurements (Table 2). Since the length of TreeQSM branches was overestimated (Table 1), our TreeQSM $\beta_{\text {branch,tree,plot were higher than the measured }}$ exponents, as in Table 3 and Figure 4 . Interestingly, the radii of the TreeQSM branches were generally underestimated (Table 1) and our TreeQSM $\alpha_{\text {branch }}$ were therefore lower than the measured exponents as in Table 3 and Figure 4. As discussed in Lau et al. (2018), a correct definition of the branch measurements is essential to avoid ambiguity and lower absolute errors. 


\subsection{Reducing uncertainties in the branch reconstruction}

One strategy to reduce error in model estimations is to improve the point cloud quality. Tree architecture relies on hardwood measurements and the presence of leaves introduces uncertainty in the derived branch length and branch radius, which leads to uncertainty in the scaling exponent estimation. To reconstruct the small, higher order branches, we need to defoliate the tree point cloud. At the time the current analysis was carried out, no algorithm to digitally remove leaves was available. However, the leaf/wood separation algorithm from Vicari (2017) is a promising tool for future research. Achieving a point cloud density sufficient enough for modelling small branches in-situ is challenging (Lau et al. 2018; Wilkes et al., 2017). In this study, we used a scan resolution of $0.06^{\circ}$. As mentioned in Wilkes et al. (2017), increasing the resolution to $0.04^{\circ}$ would increase the point density in the point cloud (especially on the top of the canopy), making TreeQSM tree models more representative of the actual tree. Another strategy to reduce divergence between models and the field measured estimates might be to use another tree parameter that is easier to obtain from the tree point cloud, with the proviso that this parameter can be linked to plant scaling models. Instead of branch length and radius, several studies used the biomass to relate architecture to metabolic scaling (Muller-Landau et al., 2006, Nygren and Pallardy, 2008). Tree above-ground biomass can be estimated from tree point clouds of tropical trees with good concordance with reference estimations (Calders et al., 2015, Gonzalez de Tanago et al., 2017).

\subsection{Do small branches can be representative of a whole tree?}

Since using TLS to analyse branch architecture represents a significant cost and time investment, it is important to determine if our approach needs to be applied to an entire tree to determine accurate scaling exponents. A pattern can be observed in the cumulative scaling exponents between the TreeQSM and the manually measured branches (Fig. 4 and Table A.2 in Appendix). While $\alpha_{\text {branch }}$ and $\theta_{\text {branch }}$ had no substantial variation with cumulative branch order, $\beta_{\text {branch }}$ had a high (pseudo) median in the first two cumulative branch orders and then decreased from the $4^{\text {th }}$ cumulative branch order. We theorize that $\beta$ ratio is high at these first two cumulative branch orders due to the ratio between the length of parent branch (in this case, the main stem) and the length of daughter branches. This difference can be up to several meters, having a direct effect on $\beta_{\text {branch }}$ at this cumulative branch order. Having a stable pattern in higher branch orders opens the possibility that an analysis of the whole tree might be not needed to estimate scaling exponents; instead, the higher order branching section could potentially be used to estimate the tree scaling exponent for the whole tree. However, due to the lack of sufficient observations in higher branch orders, we cannot verify this hypothesis in this study. We suggest future studies to increase the branch sampling in higher branch orders to test our hypothesis.

\subsection{WBE scaling exponents from 3D tree modelling of tropical trees}

Our results of the scaling exponents from the tropical trees assessed in this study were not in concordance with the WBE predicted exponents for the scaling of $\alpha, \beta$ and $\theta$. Moreover, this study found out that while TreeQSM $\alpha_{\text {branch,tree }}$ were relatively close to the theoretical value, $\beta_{\text {branch,tree }}$

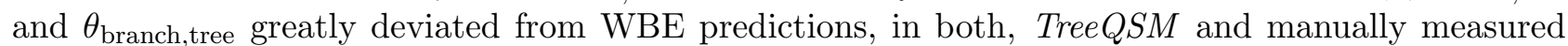
datasets.

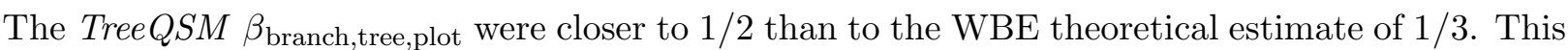
finding is consistent with Bentley et al. (2013) and Muller-Landau et al. (2006), who also observed that observed exponents significantly differed from predicted theoretical exponents. Bentley et al. (2013) suggested that $\beta=1 / 3$ might only occur in large trees, but our results do not support this hypothesis although our tree sample comprised trees with DBH ranging between $61.3 \mathrm{~cm}$ to $97.0 \mathrm{~cm}$. As mentioned by Malhi et al. (2018), the tree's response to the environment to maximize light capture through maximizing vertical height; maximize efficiency of resource distribution and minimize the risk 
of breakage or overturning might be the reason why trees appear more plastic in their lengths than in their radius (Bentley et al., 2013; Price et al., 2007).

In addition to the deviation observed for $\beta$; our findings for TreeQSM $\theta_{\text {branch,tree were lower than }}$ WBE prediction of 0.69. Metabolic rate is directly calculated from $\alpha$ and $\beta$ exponents. Since both exponents showed deviation, it was expected that metabolic rate would also differ from theoretical predictions. Our results are more aligned with those by Savage et al. (2010); Sperry et al. (2012); von Allmen et al. (2012); Bentley et al. (2013); Smith et al. (2014b), whose estimates all deviated (with lower exponents) from predicted exponents. Our results at branch-level and tree-level were on average closer to 0.69 metabolic rate than the original 3/4 proposed by WBE.

Fractal branching and homogeneous length and diameter parameters within the same branch node were not found in our dataset. As mentioned by Petit and Anfodillo (2009), the fractal branching proposed by the WBE model is very unlikely to be found in real plants. The scaling exponents deviated substantially from the exponents predicted from symmetrical and self-similar branches as proposed by the WBE model. The WBE predictions might work on individual trees which grow in absence of competition and no nutrition limitation, such as on plantations (Muller-Landau et al., 2006); or might work on young trees with simple branching rules (Petit and Anfodillo, 2009; Loehle, 2016). Those trees would have enough nutrients and would be protected from environmental hazards (such as heavy wind or rainfall) and with small branch size distributions which might be easily measured. Our sample trees do not fall into those assumptions. Predictions for large trees, as explained by Loehle (2016), are still puzzling due to the architectural complexity of real trees, their susceptibility to damage and their rapid resilience; characteristics unfitted for the symmetrical branching geometry proposed by WBE model.

Our sample also showed large dispersion around the pseudo-median exponents supporting Loehle's statement which says that optimal branching cannot be found in old trees or with increased exposure to the environment. We suggest a further study using the current methodology for WBE predictions from asymmetric branching (Brummer et al., 2017) and TreeQSM to estimate $\theta$. As suggested by Smith et al. (2014b); Price et al. (2009); Brummer et al. (2017), the theoretical value for metabolic scaling in the WBE context might be more an approximate rather than an exact value when applied to real trees.

\section{Conclusions}

We present a novel approach based on Terrestrial Laser Scanning (TLS) to measure branch architecture traits and so estimate scaling exponents within the context of the West, Brown and Enquist (WBE) model. We also manually measured the branch architecture for nine large trees in Guyana and tested both the model and theory estimates of scaling exponents against those derived from field data. The consistent biases found between the TreeQSM and the manually measured exponents showed that the bias found on the measured branching architecture influenced the estimations of the $\alpha$ and $\beta$ scaling exponents, and thus, the computation of $\theta$ exponent. The manually measured $\alpha$ and $\beta$ scaling exponents diverged from the WBE's theoretical exponents at branch-level, cumulative branch orders and tree-level suggesting that trees in tropical environments might not follow the predictions for the symmetrical branching geometry proposed by the WBE. The TreeQSM scaling exponents predicted similar architecture-based metabolic rate exponents as the manually measured exponents, although this was due to the combination of $\alpha$ and $\beta$ scaling exponents which were both biased. In particular the TreeQSM and the manually measured estimates converged at branch-level, cumulative branch order and tree-level; but diverged at plot-level. More tests are needed to validate this methodology as a consolidated approach to account for individual tree structure and as a provider of enough detailed architectural information to estimate scaling exponents at branch-, tree-, and plot-level in forested ecosystems. This study identifies a much easier way to generate data for plant scaling models using large datasets collected non-destructively in the field, rather than with the smaller datasets obtained from tedious and time-consuming hand-collection of data. Moreover, this approach could 
potentially be linked to LiDAR systems mounted on Unnamed Aerial Vehicle (UAV) may provide valuable information on top-of-the-canopy branches that are not well described from the ground (ie. using TLS) - or on the entire vertical profile when the forest canopy is not too closed (Brede et al. 2017) and could potentially be implemented for large-scale plant assessments at a regional or global scale (Bazezew et al., 2018).

\section{Author's Contribution}

$\mathrm{AL}$ coordinated the entire study; $\mathrm{AL}$ and $\mathrm{LPB}$ conceived the idea and designed the methodology; $\mathrm{AL}$ collected the data; AL, LPB and HB analysed the data; AL wrote the manuscript. HB, CM, AS, MH, TJ, YM, and LPB contributed and edited the drafts. All authors gave final approval for publication and declare no conflict of interest.

\section{Funding sources}

This research is part of CIFOR's Global Comparative Study on REDD+. The funding partners whose support is greatly appreciated include the Norwegian Agency for Development Cooperation (Norad), the Australian Department of Foreign Affairs and Trade (DFAT), the European Union (EU), the International Climate Initiative (IKI) of the German Federal Ministry for the Environment, Nature Conservation, Building and Nuclear Safety (BMUB), the CGIAR Research Program on Forests, Trees and Agroforestry (CRP-FTA) with financial support from the donors to the CGIAR Fund and ERAGAS NWO-3DforMod project 5160957540. LPB, AS and YM were supported by an ERC Advanced Investigator Award to YM (GEM-TRAITS, 321131) and NERC grant NE/P012337/1.

\section{Acknowledgements}

We acknowledge Pasi Raumonen and the collaboration of Guyana Forestry Commission for their support: Pradeepa Bholanath, Carey Bhojedat, Hansrajie Sukhdeo and their team who assisted us with logistics and during the tree harvesting in Guyana.

\section{Research data}

The individual trees TLS point clouds, QSM cylinder models, and destructive sampling measurement data used for this research can be accessed via the 4TU.Center for Research Data Repository (DOI: 10.4121/uuid:0120f3c6-cfa6-42a5-84bf-d9e598283c59). These datasets are owned by the CIFOR and Wageningen University. The datasets are free to download and available for any use as long as the proper reference, as specified in the portal, is applied. For collaborations or questions please contact the corresponding author.

\section{Appendix A}

\section{A.1 WBE metabolic scaling exponents}

The WBE theory holds that the scaling of metabolic rate and other biological functions has its origin in an optimal branching system network at both internal (vascular) and external (branching) components (West et al., 1997; West, 1999). While the internal structure is composed by xylem and phloem conduits, the external structure is composed by branches. The WBE theory assumes that an idealized external tree branching network is symmetrical, self-similar and hierarchical (see Figure 3 in Malhi et al. (2018)), organized in such a way that metabolic rate should not vary when comparing branch node-level to the whole tree-level (West, 1999; Nygren and Pallardy, 2008; Sperry et al., 2012, 
Bentley et al., 2013). Nevertheless, real trees do not show an idealized external branching network. Self-similarity rarely holds true throughout a whole tree, branch order varies across tree-level and stems taper and exhibit asymmetric branching (Nygren and Pallardy, 2008; Smith et al., 2014b; Price et al., 2012; Bentley et al., 2013).
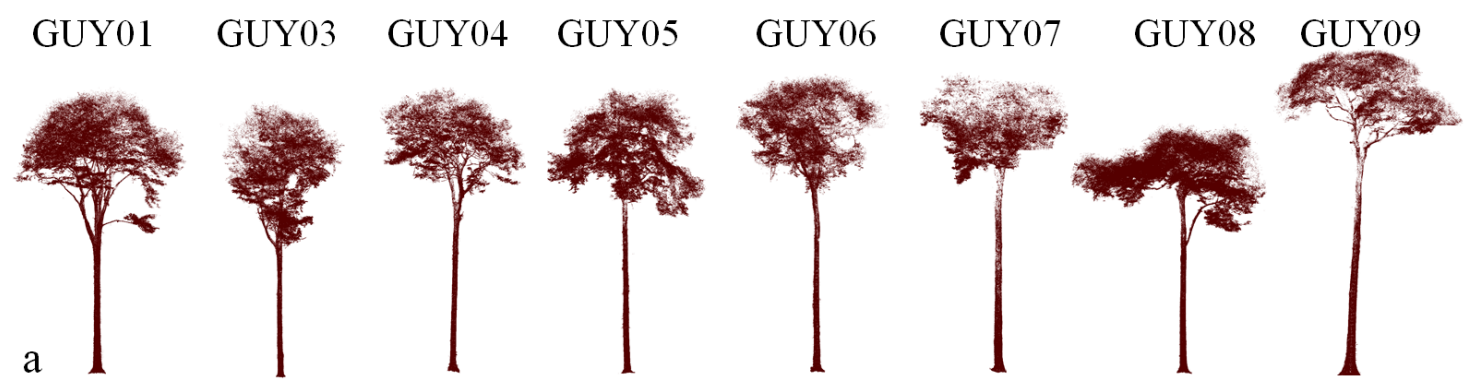

GUY10
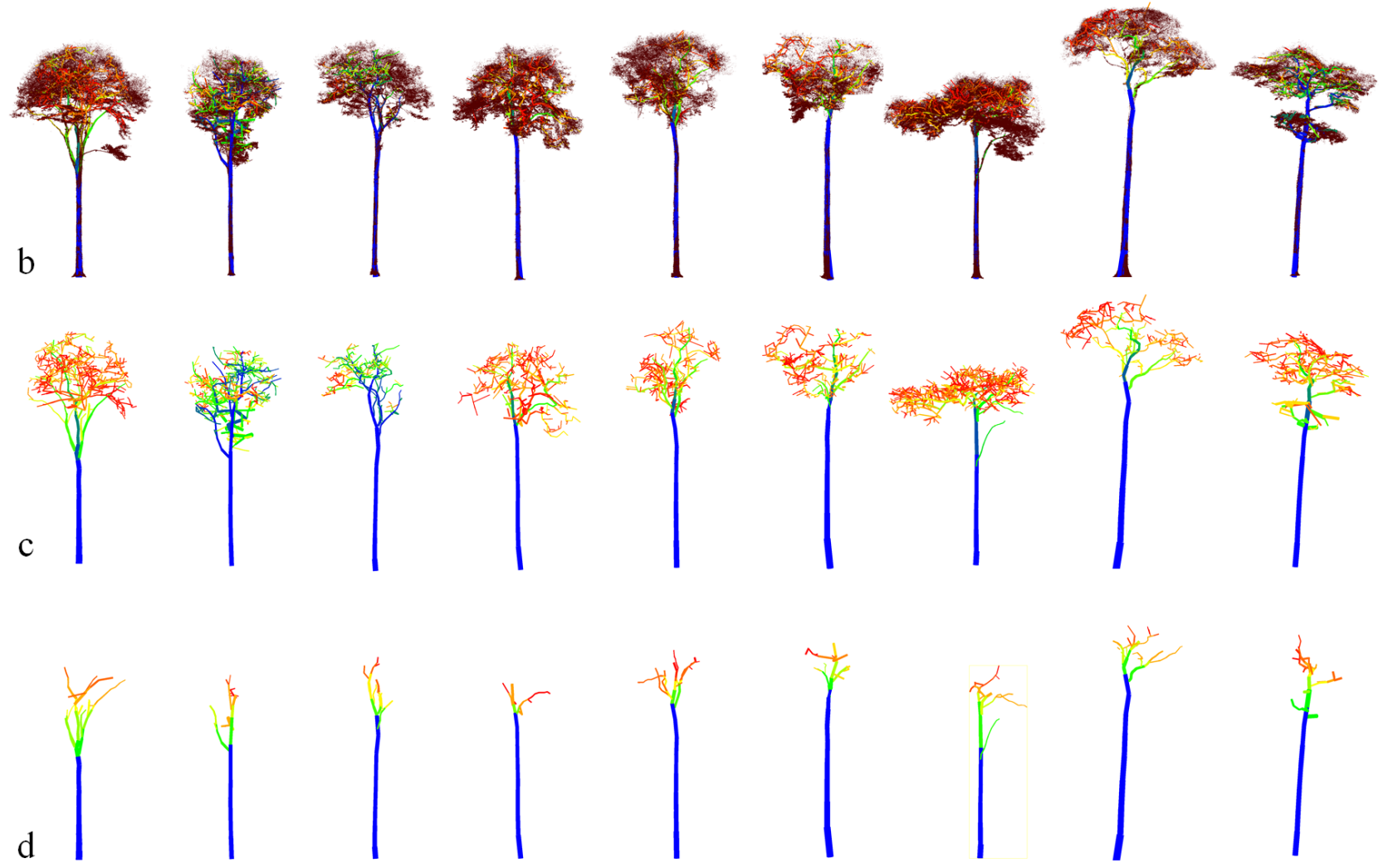

Figure A.1: Tree point clouds and TreeQSM models from the nine trees scanned in Guyana. (a) tree point clouds of the nine trees from Guyana, (b) One repetition of TreeQSM with branches $>10 \mathrm{~cm}$ diameter reconstructed along with the tree point clouds, (c) QSM branches classified by branch order, and (d) QSM branches which were paired with manually measured branches. GUY01 to GUY08 are Eperua grandiflora trees, GUY09 is a Ormosia coutinhoi tree, and GUY10 is a Eperua falcata tree. 
Table A.1: Normality test (Shapiro-Wilks) for TreeQSM and manually measured scaling exponents at branch-level $(\underline{p \text {-value }<0.05)}$.

\begin{tabular}{clc}
\hline \multirow{2}{*}{ Exponent type } & \multicolumn{2}{c}{$p$-value } \\
\cline { 2 - 3 } & \multicolumn{1}{c}{ TreeQSM } & Measured \\
\hline Radius scaling - $\alpha$ & 0.03 & $1.73 \times 10^{-9}$ \\
Length scaling - $\beta$ & 0.02 & $4.23 \times 10^{-5}$ \\
Architecture-based metabolic rate $-\theta$ & $1.38 \times 10^{-41}$ & $9.05 \times 10^{-42}$ \\
\hline
\end{tabular}

Table A.2: (pseudo) median, CI (95\%), bias (\%), and significance for $\alpha_{\text {branch }}, \beta_{\text {branch }}$ and $\theta_{\text {branch }}$ for cumulative branch order for TreeQSM and manually measured branches.

\begin{tabular}{|c|c|c|c|c|c|c|c|}
\hline \multirow{3}{*}{$\begin{array}{l}\text { Cumulative } \\
\text { branch order }\end{array}$} & \multirow{3}{*}{$\begin{array}{c}\text { Number } \\
\text { of } \\
\text { obs. }\end{array}$} & \multicolumn{6}{|c|}{ Radius scaling exponent - $\alpha$} \\
\hline & & \multicolumn{2}{|c|}{ TreeQSM } & \multicolumn{2}{|c|}{ Measured } & \multirow{2}{*}{ Bias (\%) } & \multirow{2}{*}{ Signif. } \\
\hline & & (pseudo) & CI $(95 \%)$ & (pseudo) & CI $(95 \%)$ & & \\
\hline up to 2 nd & 63 & 0.70 & $0.63-0.75$ & 0.61 & $0.57-0.65$ & 15 & $\dagger$ \\
\hline up to $3 \mathrm{rd}$ & 184 & 0.54 & $0.50-0.58$ & 0.64 & $0.59-0.66$ & -15 & $*$ \\
\hline up to 4 th & 330 & 0.51 & $0.48-0.54$ & 0.64 & $0.62-0.66$ & -20 & $*$ \\
\hline up to 5 th & 422 & 0.48 & $0.45-0.50$ & 0.64 & $0.62-0.66$ & -20 & * \\
\hline up to 6 th & 467 & 0.46 & $0.44-0.48$ & 0.64 & $0.62-0.66$ & -28 & * \\
\hline up to 7 th & 482 & 0.46 & $0.43-0.48$ & 0.63 & $0.62-0.65$ & -28 & $*$ \\
\hline up to 8 th & 484 & 0.45 & $0.43-0.48$ & 0.63 & $0.62-0.65$ & -28 & $*$ \\
\hline
\end{tabular}

* significant different at 0.05 probability level.

$\dagger$ non significant different at 0.05 probability level.

Table A.2: Extended

\begin{tabular}{|c|c|c|c|c|c|c|c|}
\hline \multirow{3}{*}{$\begin{array}{l}\text { Cumulative } \\
\text { branch order }\end{array}$} & \multirow{3}{*}{$\begin{array}{c}\text { Number } \\
\text { of } \\
\text { obs. }\end{array}$} & \multicolumn{6}{|c|}{ Length scaling exponent - $\beta$} \\
\hline & & \multicolumn{2}{|c|}{ TreeQSM } & \multicolumn{2}{|c|}{ Measured } & \multirow[t]{2}{*}{ Bias (\%) } & \multirow[t]{2}{*}{ Signif. } \\
\hline & & $\begin{array}{l}\text { (pseudo) } \\
\text { median }\end{array}$ & CI $(95 \%)$ & $\begin{array}{l}\text { (pseudo) } \\
\text { median }\end{array}$ & CI $(95 \%)$ & & \\
\hline up to 2 nd & 63 & 2.40 & $2.22-2.63$ & 2.27 & $1.98-2.46$ & 6 & $\dagger$ \\
\hline up to $3 \mathrm{rd}$ & 184 & 1.01 & $0.80-1.23$ & 0.84 & $0.59-1.03$ & 21 & $\dagger$ \\
\hline up to 4 th & 330 & 0.60 & $0.46-0.75$ & 0.32 & $0.17-0.47$ & 91 & $*$ \\
\hline up to 5 th & 422 & 0.48 & $0.36-0.61$ & 0.07 & $-0.06-0.22$ & 549 & $*$ \\
\hline up to 6 th & 467 & 0.44 & $0.32-0.56$ & 0.11 & $-0.01-0.26$ & 282 & $*$ \\
\hline up to 7 th & 482 & 0.43 & $0.31-0.54$ & 0.07 & $-0.06-0.20$ & 476 & $*$ \\
\hline up to 8 th & 484 & 0.42 & $0.31-0.54$ & 0.07 & $-0.06-0.20$ & 497 & $*$ \\
\hline
\end{tabular}


Table A.2: Extended

\begin{tabular}{|c|c|c|c|c|c|c|c|}
\hline \multirow{3}{*}{$\begin{array}{l}\text { Cumulative } \\
\text { branch order }\end{array}$} & \multirow{3}{*}{$\begin{array}{c}\text { Number } \\
\text { of } \\
\text { obs. }\end{array}$} & \multicolumn{6}{|c|}{ Architecture-based metabolic rate $-\theta$} \\
\hline & & \multicolumn{2}{|c|}{ TreeQSM } & \multicolumn{2}{|c|}{ Measured } & \multirow[t]{2}{*}{ Bias (\%) } & \multirow[t]{2}{*}{ Signif. } \\
\hline & & $\begin{array}{c}\text { (pseudo) } \\
\text { median }\end{array}$ & CI $(95 \%)$ & $\begin{array}{c}\text { (pseudo) } \\
\text { median }\end{array}$ & CI $(95 \%)$ & & \\
\hline up to 2 nd & 63 & 0.28 & $0.26-0.29$ & 0.30 & $0.28-0.31$ & -9 & $\dagger$ \\
\hline up to $3 \mathrm{rd}$ & 184 & 0.47 & $0.39-0.56$ & 0.47 & $0.43-0.53$ & 0 & $\dagger$ \\
\hline up to 4 th & 330 & 0.56 & $0.50-0.62$ & 0.58 & $0.52-0.65$ & -3 & $\dagger$ \\
\hline up to 5 th & 422 & 0.58 & $0.52-0.65$ & 0.51 & $0.45-0.58$ & 14 & $\dagger$ \\
\hline up to 6 th & 467 & 0.58 & $0.51-0.64$ & 0.50 & $0.44-0.56$ & 16 & $\dagger$ \\
\hline up to 7 th & 482 & 0.58 & $0.52-0.65$ & 0.50 & $0.44-0.56$ & 17 & $\dagger$ \\
\hline up to 8 th & 484 & 0.58 & $0.52-0.65$ & 0.50 & $0.44-0.57$ & 16 & $\dagger$ \\
\hline
\end{tabular}

Table A.3: (pseudo) median exponents, 95\% CI, bias (\%), and significance for $\alpha_{\text {tree }}, \beta_{\text {tree }}$ and $\theta_{\text {tree }}$ per individual tree and plot-level (pseudo) median ranges among trees.

\begin{tabular}{|c|c|c|c|c|c|c|c|}
\hline \multirow{4}{*}{ Tree } & \multirow{4}{*}{$\begin{array}{c}\text { Number } \\
\text { of } \\
\text { obs. }\end{array}$} & \multicolumn{6}{|c|}{ Radius scaling exponent $-\alpha$} \\
\hline & & \multicolumn{2}{|c|}{ TreeQSM } & \multicolumn{2}{|c|}{ Measured } & \multirow[t]{3}{*}{ Bias (\%) } & \multirow[t]{3}{*}{ Signif. } \\
\hline & & (pseudo) & CI $(95 \%)$ & (pseudo) & CI $(95 \%)$ & & \\
\hline & & median & & median & & & \\
\hline GUY01 & 64 & 0.49 & $0.44-0.54$ & 0.66 & $0.61-0.70$ & -26 & $*$ \\
\hline GUY03 & 50 & 0.45 & $0.36-0.52$ & 0.62 & $0.60-0.69$ & -29 & $*$ \\
\hline GUY04 & 48 & 0.43 & $0.37-0.49$ & 0.69 & $0.65-0.76$ & -37 & $*$ \\
\hline GUY05 & 38 & 0.44 & $0.32-0.56$ & 0.68 & $0.65-0.75$ & -35 & $*$ \\
\hline GUY06 & 52 & 0.47 & $0.41-0.51$ & 0.62 & $0.54-0.67$ & -25 & $*$ \\
\hline GUY07 & 50 & 0.5 & $0.40-0.60$ & 0.65 & $0.62-0.68$ & -23 & $*$ \\
\hline GUY08 & 50 & 0.5 & $0.41-0.61$ & 0.66 & $0.6-0.73$ & -23 & $*$ \\
\hline GUY09 & 65 & 0.52 & $0.46-0.59$ & 0.62 & $0.56-0.66$ & -15 & $*$ \\
\hline GUY10 & 67 & 0.33 & $0.28-0.38$ & 0.53 & $0.46-0.59$ & -38 & $*$ \\
\hline plot-level & 10 & $0.33-0.52$ & NA & $0.53-0.69$ & NA & NA & NA \\
\hline
\end{tabular}

* significant at 0.05 probability level.

$\dagger$ non significant at 0.05 probability level.

NA not available. 
Table A.3: Extended

\begin{tabular}{|c|c|c|c|c|c|c|c|}
\hline \multirow{4}{*}{ Tree } & \multirow{4}{*}{$\begin{array}{c}\text { Number } \\
\text { of } \\
\text { obs. }\end{array}$} & \multicolumn{6}{|c|}{ Length scaling exponent - $\beta$} \\
\hline & & \multicolumn{2}{|c|}{ TreeQSM } & \multicolumn{2}{|c|}{ Measured } & \multirow[t]{3}{*}{ Bias (\%) } & \multirow[t]{3}{*}{ Signif. } \\
\hline & & (pseudo) & CI $(95 \%)$ & (pseudo) & CI (95\%) & & \\
\hline & & median & & median & & & \\
\hline GUY01 & 64 & 0.21 & $-0.06-0.60$ & -0.12 & $-0.32-0.25$ & -281 & $\dagger$ \\
\hline GUY03 & 50 & 0.59 & $0.24-0.99$ & 0.3 & $0.10-0.49$ & 97 & $\dagger$ \\
\hline GUY04 & 48 & 0.35 & $-0.04-0.79$ & 0.13 & $-0.23-0.42$ & 164 & $\dagger$ \\
\hline GUY05 & 38 & 0.17 & $-0.31-1.04$ & -0.33 & $-0.71-0.59$ & -153 & $\dagger$ \\
\hline GUY06 & 52 & 0.55 & $0.10-0.99$ & 0.42 & $-0.11-0.99$ & 29 & $\dagger$ \\
\hline GUY07 & 50 & 0.62 & $0.24-1.08$ & -0.19 & $-0.53-0.30$ & -426 & $*$ \\
\hline GUY08 & 50 & 0.44 & $0.12-0.74$ & 0.28 & $-0.20-0.75$ & 57 & $\dagger$ \\
\hline GUY09 & 65 & 0.57 & $0.26-0.83$ & 0.05 & $-0.35-0.38$ & 1027 & $*$ \\
\hline GUY10 & 67 & 0.23 & $0 \quad-0.5$ & -0.11 & $-0.51-0.32$ & -302 & $\dagger$ \\
\hline plot-level & 10 & $0.17-0.62$ & NA & $-0.33-0.42$ & NA & NA & NA \\
\hline
\end{tabular}

Table A.3: Extended

\begin{tabular}{|c|c|c|c|c|c|c|c|}
\hline \multirow{3}{*}{ Tree } & \multirow{3}{*}{$\begin{array}{c}\text { Number } \\
\text { of } \\
\text { obs. }\end{array}$} & \multicolumn{6}{|c|}{ Architecture-based metabolic rate - $\theta$} \\
\hline & & \multicolumn{2}{|c|}{ TreeQSM } & \multicolumn{2}{|c|}{ Measured } & \multirow[t]{2}{*}{ Bias (\%) } & \multirow[t]{2}{*}{ Signif. } \\
\hline & & $\begin{array}{l}\text { (pseudo) } \\
\text { median }\end{array}$ & CI $(95 \%)$ & $\begin{array}{l}\text { (pseudo) } \\
\text { median }\end{array}$ & CI $(95 \%)$ & & \\
\hline GUY01 & 64 & 0.69 & $0.45-0.88$ & 0.52 & $0.39-0.65$ & 33 & $\dagger$ \\
\hline GUY03 & 50 & 0.56 & $0.38-0.84$ & 0.64 & $0.53-0.76$ & -12 & $\dagger$ \\
\hline GUY04 & 48 & 0.38 & $-0.27-0.56$ & 0.47 & $0.41-0.59$ & -20 & $\dagger$ \\
\hline GUY05 & 38 & 0.24 & $-0.39-0.65$ & 0.38 & $-1.26-0.69$ & -36 & $\dagger$ \\
\hline GUY06 & 52 & 0.51 & $0.34-0.84$ & 0.38 & $0.23-0.92$ & 35 & $\dagger$ \\
\hline GUY07 & 50 & 0.75 & $0.55-1.04$ & 0.76 & $0.58-0.97$ & -1 & $\dagger$ \\
\hline GUY08 & 50 & 0.53 & $0.37-0.67$ & 0.27 & $-2.18-0.34$ & 101 & $*$ \\
\hline GUY09 & 65 & 0.59 & $0.48-0.83$ & 0.71 & $0.54-0.96$ & -16 & $\dagger$ \\
\hline GUY10 & 67 & 0.77 & $0.57-1.02$ & 0.45 & $0.25-0.95$ & 72 & $\dagger$ \\
\hline plot-level & 10 & $0.24-0.77$ & NA & $0.27-0.76$ & NA & NA & NA \\
\hline
\end{tabular}

\section{Appendix B. Supplementary material}

Supplementary data associated with this article can be found, in the online version, at https://doi. org $/ 10.1016 / j$.foreco.2019.02.019.

\section{References}

Åkerblom, M., Raumonen, P., Mäkipää, R., and Kaasalainen, M. (2017). Automatic tree species recognition with quantitative structure models. Remote Sensing of Environment, 191:1-12.

Ayrey, E., Fraver, S., Kershaw, J. A., Kenefic, L. S., Hayes, D., Weiskittel, A. R., and Roth, B. E. (2017). Layer Stacking: A Novel Algorithm for Individual Forest Tree Segmentation from LiDAR Point Clouds. Canadian Journal of Remote Sensing, 43(1):16-27.

Bazezew, M. N., Hussin, Y. A., and Kloosterman, E. (2018). Integrating Airborne LiDAR and Terrestrial Laser Scanner forest parameters for accurate above-ground biomass/carbon estimation in 
Ayer Hitam tropical forest, Malaysia. International Journal of Applied Earth Observation and Geoinformation, 73(June 2017):638-652.

Bentley, L. P., Stegen, J. C., Savage, V. M., Smith, D. D., von Allmen, E. I., Sperry, J. S., Reich, P. B., and Enquist, B. J. (2013). An empirical assessment of tree branching networks and implications for plant allometric scaling models. Ecology Letters, 16(8):1069-1078.

Brede, B., Lau, A., Bartholomeus, H., and Kooistra, L. (2017). Comparing RIEGL RiCOPTER UAV LiDAR Derived Canopy Height and DBH with Terrestrial LiDAR. Sensors, 17(10):2371.

Brummer, A. B., Savage, V. M., and Enquist, B. J. (2017). A general model for metabolic scaling in self-similar asymmetric networks. PLoS Computational Biology, 13(3):1-25.

Burt, A., Disney, M., Raumonen, P., Armston, J., Calders, K., and Lewis, P. (2013). Rapid characterisation of forest structure from TLS and 3D modelling. In 2013 IEEE International Geoscience and Remote Sensing Symposium - IGARSS, number 128.197.168.195, pages 3387-3390. IEEE.

Calders, K., Newnham, G. G., Burt, A., Murphy, S., Raumonen, P., Herold, M., Culvenor, D., Avitabile, V., Disney, M., Armston, J., and Kaasalainen, M. (2015). Nondestructive estimates of above-ground biomass using terrestrial laser scanning. Methods in Ecology and Evolution, 6(2):198208.

Côté, J.-F., Fournier, R. A., Frazer, G. W., and Olaf Niemann, K. (2012). A fine-scale architectural model of trees to enhance LiDAR-derived measurements of forest canopy structure. Agricultural and Forest Meteorology, 166-167:72-85.

Dassot, M., Constant, T., and Fournier, M. (2011). The use of terrestrial LiDAR technology in forest science: application fields, benefits and challenges. Annals of Forest Science, 68(5):959-974.

Gonzalez de Tanago, J., Lau, A., Bartholomeus, H., Herold, M., Avitabile, V., Raumonen, P., Martius, C., Goodman, R. C., Disney, M., Manuri, S., Burt, A., and Calders, K. (2017). Estimation of aboveground biomass of large tropical trees with terrestrial LiDAR. Methods in Ecology and Evolution, 2017(July):1-12.

Grau, E., Durrieu, S., Fournier, R., Gastellu-Etchegorry, J.-P., and Yin, T. (2017). Estimation of $3 \mathrm{D}$ vegetation density with Terrestrial Laser Scanning data using voxels. A sensitivity analysis of influencing parameters. Remote Sensing of Environment, 191:373-388.

Hackenberg, J., Spiecker, H., Calders, K., Disney, M., and Raumonen, P. (2015). SimpleTree - An Efficient Open Source Tool to Build Tree Models from TLS Clouds. Forests, 6(12):4245-4294.

Hallé, F., Oldeman, R. A. A., and Tomlinson, P. B. (1978). Tropical Trees and Forests. Springer Berlin Heidelberg, Berlin, Heidelberg.

Huang, H., Li, Z., Gong, P., Cheng, X., Clinton, N., Cao, C., Ni, W., and Wang, L. (2011). Automated Methods for Measuring DBH and Tree Heights with a Commercial Scanning Lidar. Photogrammetric Engineering \& Remote Sensing, 77(3):219-227.

Kaasalainen, S., Krooks, A., Liski, J., Raumonen, P., Kaartinen, H., Kaasalainen, M., Puttonen, E., Anttila, K., and Mäkipää, R. (2014). Change detection of tree biomass with terrestrial laser scanning and quantitative structure modelling. Remote Sensing, 6(5):3906-3922.

Krooks, A., Kaasalainen, S., Kankare, V., Joensuu, M., Raumonen, P., and Kaasalainen, M. (2014). Tree structure vs. height from terrestrial laser scanning and quantitative structure models. Silva Fennica, 48(2):1-11. 
Lau, A., Bentley, L. P., Martius, C., Shenkin, A., Bartholomeus, H., Raumonen, P., Malhi, Y., Jackson, T., and Herold, M. (2018). Quantifying branch architecture of tropical trees using terrestrial LiDAR and 3D modelling. Trees - Structure and Function, page 13.

Loehle, C. (2016). Biomechanical constraints on tree architecture. Trees, 30(6):2061-2070.

Malhi, Y., Jackson, T., Patrick Bentley, L., Lau, A., Shenkin, A., Herold, M., Calders, K., Bartholomeus, H., and Disney, M. I. (2018). New perspectives on the ecology of tree structure and tree communities through terrestrial laser scanning. Interface Focus, 8(2):20170052.

McMahon, T. A. and Kronauer, R. E. (1976). Tree structures: deducing the principle of mechanical design. Journal of theoretical biology, 59:443-466.

Momo Takoudjou, S., Ploton, P., Sonké, B., Hackenberg, J., Griffon, S., de Coligny, F., Kamdem, N. G., Libalah, M., Mofack, G. I. I., Le Moguédec, G., Pélissier, R., and Barbier, N. (2018). Using terrestrial laser scanning data to estimate large tropical trees biomass and calibrate allometric models: A comparison with traditional destructive approach. Methods in Ecology and Evolution, 9(4):905-916.

Muller-Landau, H. C., Condit, R. S., Chave, J., Thomas, S. C., Bohlman, S. A., Bunyavejchewin, S., Davies, S., Foster, R., Gunatilleke, S., Gunatilleke, N., Harms, K. E., Hart, T., Hubbell, S. P., Itoh, A., Kassim, A. R., LaFrankie, J. V., Lee, H. S., Losos, E., Makana, J.-R., Ohkubo, T., Sukumar, R., Sun, I.-F., Nur Supardi, M. N., Tan, S., Thompson, J., Valencia, R., Munoz, G. V., Wills, C., Yamakura, T., Chuyong, G., Dattaraja, H. S., Esufali, S., Hall, P., Hernandez, C., Kenfack, D., Kiratiprayoon, S., Suresh, H. S., Thomas, D., Vallejo, M. I., and Ashton, P. (2006). Testing metabolic ecology theory for allometric scaling of tree size, growth and mortality in tropical forests. Ecology Letters, 9(5):575-588.

Newnham, G. G., Armston, J. D., Calders, K., Disney, M. I., Lovell, J. L., Schaaf, C. B., Strahler, A. H., and Danson, F. M. (2015). Terrestrial Laser Scanning for Plot-Scale Forest Measurement. Current Forestry Reports, 1(4):239-251.

Niklas, K. J. (1994). Plant Allometry: The Scaling of Form and Process. University of Chicago Press., Chicago.

Nygren, P. and Pallardy, S. G. (2008). Applying a universal scaling model to vascular allometry in a single-stemmed, monopodially branching deciduous tree (Attim's model). Tree physiology, 28(1):1-10.

Olagoke, A., Proisy, C., Féret, J.-B., Blanchard, E., Fromard, F., Mehlig, U., de Menezes, M. M., dos Santos, V. F., and Berger, U. (2016). Extended biomass allometric equations for large mangrove trees from terrestrial LiDAR data. Trees, 30(3):935-947.

Olivier, M.-D. D., Robert, S., and Richard A., F. (2017). A method to quantify canopy changes using multi-temporal terrestrial lidar data: Tree response to surrounding gaps. Agricultural and Forest Meteorology, 237-238:184-195.

Paynter, I., Saenz, E., Genest, D., Peri, F., Erb, A., Li, Z., Wiggin, K., Muir, J., Raumonen, P., Schaaf, E. S., Strahler, A., and Schaaf, C. (2016). Observing ecosystems with lightweight, rapid-scanning terrestrial lidar scanners. Remote Sensing in Ecology and Conservation, 2(4):174-189.

Petit, G. and Anfodillo, T. (2009). Plant physiology in theory and practice: An analysis of the WBE model for vascular plants. Journal of Theoretical Biology, 259:1-4.

Price, C. A., Enquist, B. J., and Savage, V. M. (2007). A general model for allometric covariation in botanical form and function. Proceedings of the National Academy of Sciences, 104(32):13204-13209. 
Price, C. A., Ogle, K., White, E. P., and Weitz, J. S. (2009). Evaluating scaling models in biology using hierarchical Bayesian approaches. Ecology Letters, 12(7):641-651.

Price, C. a., Weitz, J. S., Savage, V. M., Stegen, J., Clarke, A., Coomes, D. a., Dodds, P. S., Etienne, R. S., Kerkhoff, A. J., McCulloh, K., Niklas, K. J., Olff, H., and Swenson, N. G. (2012). Testing the metabolic theory of ecology. Ecology Letters, 15(12):1465-1474.

Raumonen, P., Casella, E., Calders, K., Murphy, S., Åkerblom, M., and Kaasalainen, M. (2015). MASSIVE-SCALE TREE MODELLING FROM TLS DATA. ISPRS Annals of Photogrammetry, Remote Sensing and Spatial Information Sciences, II-3/W4(March):189-196.

Raumonen, P., Kaasalainen, M., Åkerblom, M., Kaasalainen, S., Kaartinen, H., Vastaranta, M., Holopainen, M., Disney, M., and Lewis, P. (2013). Fast Automatic Precision Tree Models from Terrestrial Laser Scanner Data. Remote Sensing, 5(2):491-520.

Rosell, J., Lloyd, J., Sanz, R., Arnó, J., Ribes-Dasi, M., Masip, J., Escolà, A., Camp, F., Solanelles, F., Gràcia, F., Gil, E., Val, L., Planas, S., and Palacín, J. (2009). Obtaining the three-dimensional structure of tree orchards from remote 2D terrestrial LIDAR scanning. Agricultural and Forest Meteorology, 149(9):1505-1515.

Saarinen, N., Kankare, V., Vastaranta, M., Luoma, V., Pyörälä, J., Tanhuanpää, T., Liang, X., Kaartinen, H., Kukko, A., Jaakkola, A., Yu, X., Holopainen, M., and Hyyppä, J. (2017). Feasibility of Terrestrial laser scanning for collecting stem volume information from single trees. ISPRS Journal of Photogrammetry and Remote Sensing, 123:140-158.

Saatchi, S., Marlier, M., Chazdon, R. L., Clark, D. B., and Russell, A. E. (2011). Impact of spatial variability of tropical forest structure on radar estimation of aboveground biomass. Remote Sensing of Environment, 115(11):2836-2849.

Savage, V. M., Bentley, L. P., Enquist, B. J., Sperry, J. S., Smith, D. D., Reich, P. B., and von Allmen, E. I. (2010). Hydraulic trade-offs and space filling enable better predictions of vascular structure and function in plants. Proceedings of the National Academy of Sciences, 107(52):22722-22727.

Savage, V. M., Deeds, E. J., and Fontana, W. (2008). Sizing up allometric scaling theory. PLoS computational biology, 4(9):e1000171.

Smith, A., Astrup, R., Raumonen, P., Liski, J., Krooks, A., Kaasalainen, S., Åkerblom, M., and Kaasalainen, M. (2014a). Tree Root System Characterization and Volume Estimation by Terrestrial Laser Scanning and Quantitative Structure Modeling. Forests, 5(12):3274-3294.

Smith, D. D., Sperry, J. S., Enquist, B. J., Savage, V. M., McCulloh, K. A., and Bentley, L. P. (2014b). Deviation from symmetrically self-similar branching in trees predicts altered hydraulics, mechanics, light interception and metabolic scaling. New Phytologist, 201(1):217-229.

Sperry, J. S., Smith, D. D., Savage, V. M., Enquist, B. J., McCulloh, K. a., Reich, P. B., Bentley, L. P., and von Allmen, E. I. (2012). A species-level model for metabolic scaling in trees I. Exploring boundaries to scaling space within and across species. Functional Ecology, 26(5):1054-1065.

Srinivasan, S., Popescu, S., Eriksson, M., Sheridan, R., and Ku, N.-W. (2015). Terrestrial Laser Scanning as an Effective Tool to Retrieve Tree Level Height, Crown Width, and Stem Diameter. Remote Sensing, 7(2):1877-1896.

Stovall, A. E., Vorster, A. G., Anderson, R. S., Evangelista, P. H., and Shugart, H. H. (2017). Nondestructive aboveground biomass estimation of coniferous trees using terrestrial LiDAR. Remote Sensing of Environment, 200(July):31-42. 
Tansey, K., Selmes, N., Anstee, A., Tate, N. J., and Denniss, A. (2009). Estimating tree and stand variables in a Corsican Pine woodland from terrestrial laser scanner data. International Journal of Remote Sensing, 30(19):5195-5209.

Tredennick, A. T., Bentley, L. P., and Hanan, N. P. (2013). Allometric Convergence in Savanna Trees and Implications for the Use of Plant Scaling Models in Variable Ecosystems. PLoS ONE, $8(3): \mathrm{e} 58241$.

van Leeuwen, M., Coops, N., Newnham, G. G., Hilker, T., Culvenor, D., and Wulder, M. A. (2011). Stem detection and measuring DBH using terrestrial laser scanning. Silvilaser, Full proceedings, pages $1-6$.

Vicari, M. (2017). TLSeparation.

von Allmen, E. I., Sperry, J. S., Smith, D. D., Savage, V. M., Enquist, B. J., Reich, P. B., and Bentley, L. P. (2012). A species-level model for metabolic scaling of trees II. Testing in a ring- and diffuse-porous species. Functional Ecology, 26(5):1066-1076.

West, G. B. (1999). The Fourth Dimension of Life: Fractal Geometry and Allometric Scaling of Organisms. Science, 284(5420):1677-1679.

West, G. B., Brown, J. H., and Enquist, B. J. (1997). A General Model for the Origin of Allometric Scaling Laws in Biology. Science, 276(5309):122-126.

West, G. B., Enquist, B. J., and Brown, J. H. (2009). A general quantitative theory of forest structure and dynamics. Proceedings of the National Academy of Sciences of the United States of America, 106:7040-7045.

Wilkes, P., Lau, A., Disney, M., Calders, K., Burt, A., Gonzalez de Tanago, J., Bartholomeus, H., Brede, B., and Herold, M. (2017). Data acquisition considerations for Terrestrial Laser Scanning of forest plots. Remote Sensing of Environment, 196:140-153.

Xi, Z., Hopkinson, C., and Chasmer, L. (2016). Automating Plot-Level Stem Analysis from Terrestrial Laser Scanning. Forests, 7(12):252.

Zhao, F., Guo, Q., and Kelly, M. (2012). Allometric equation choice impacts lidar-based forest biomass estimates: A case study from the Sierra National Forest, CA. Agricultural and Forest Meteorology, 165:64-72. 